\title{
Antenna Gain Against Interference in CDMA Macrodiversity Systems
}

\author{
Halim Yanikomeroglu, Member, IEEE, and Elvino S. Sousa, Senior Member, IEEE
}

\begin{abstract}
In a multiantenna system, there is a potential antenna gain against interference, in addition to the diversity gain achieved against fading. It is well known that in order to attain most of the diversity gain (against fading), the antenna elements should be placed apart with a distance (many times) greater than the wavelength $=[$ speed of light $] /[$ frequency $]$ of the carrier. The results presented in this paper indicate that in order to attain most of the antenna gain (against interference) in the reverse-link of finite-bandwidth interference-limited CDMA systems, the interantenna distance should be (many times) greater than a new parameter which is defined as the chiplength $=$ [speed of light $] /$ [chip rate] of the spreading code.
\end{abstract}

Index Terms-CDMA, interference analysis, macrodiversity, multiantennas, wireless communications.

\section{INTRODUCTION}

$\mathbf{T}$ HE CDMA ${ }^{1}$ reverse-link capacity of a network of antenna elements (AEs) is investigated in [1]. It is reported there that the capacity increases linearly with the number of AEs and it is further stated that this linear gain is valid irrespective of the user ${ }^{2}$ and $\mathrm{AE}$ positions (as long as neither AEs nor users are located at the same point). ${ }^{3}$ This result is obviously very important due to its fundamental nature.

The assumptions, under which the above reported result (that the capacity can be increased linearly without a bound simply by placing more AEs anywhere in the service region) is valid, need to be investigated carefully, since such a remarkable performance return sounds, even intuitively, too good to be true in a practical system.

Paper approved by C.-L. Wang, the Editor for Modulation Detection and Equalization of the IEEE Communications Society. Manuscript received February 3, 2000; revised January 3, 2001 and July 20, 2001. This work was supported in part by a grant from the Canadian Institute for Telecommunications Research (CITR) under the NCE program of the Government of Canada. This paper was presented in part at the IEEE International Conference on Communications (ICC'00), New Orleans, LA, June 18-22, 2000, at the 6th Canadian Workshop on Information Theory (CWIT'99), Kingston, ON, Canada, June 15-18, 1999, and at the IEEE International Conference on Communications (ICC'99), Vancouver, BC, Canada, June 6-10, 1999.

$\mathrm{H}$. Yanikomeroglu is with the Broadband Communications and Wireless Systems (BCWS) Centre, Department of Systems and Computer Engineering, Carleton University, Ottawa, ON K1S 5B6, Canada (e-mail: halim@sce. carleton.ca).

E. S. Sousa is with the Department of Electrical and Computer Engineering, University of Toronto, Toronto, ON M5S 3G4, Canada (e-mail: sousa@ comm.utoronto.ca)

Publisher Item Identifier 10.1109/TCOMM.2002.801521.

\footnotetext{
${ }^{1}$ In this paper, the term CDMA is used to denote DS-CDMA.

${ }^{2}$ Throughout this paper, user refers to a wireless user.

${ }^{3}$ Less general results are given in [2] as well.
}

There are many assumptions made in [1] some of which may not be practical; it is important to note, however, that these assumptions (which correspond to logical limits) are crucial in understanding the dynamics of a CDMA multi-antenna system and in evaluating its performance upper bound. Once the upper bound is obtained, the assumptions can be removed or relaxed and the performance returns in more realistic systems can be determined.

The key condition in attaining a linear capacity gain in such a macrodiversity system is that the interference picked up by different AEs have to be uncorrelated. This condition is satisfied, irrespective of the user and AE positions, only when the spread spectrum bandwidth goes to infinity since in that case the interference behaves like white noise.

It will be shown in this paper that the consequence of a finite bandwidth, on the other hand, is the possibility of correlated interference, the severity of which would depend on the relative positions of the AEs and users. This would degrade the performance by yielding a mean output SIR (signal-to-interference ratio) which is less than the sum of the mean branch SIRs and therefore, would impose a dampening effect on the capacity increase with respect to the number of AEs used.

\section{A. CDMA Macrodiversity System Description}

We consider the reverse-link of a network of $L$ omnidirectional AEs which are physically distributed in a service region (we note that the reverse-link of a CDMA multi-antenna system is inherently different from its forward-link due to the fact that in the reverse-link performance gains can be achieved without injecting extra energy into the system). The outputs of the AEs are conveyed to a central station (CS) with separate feeders for decoding and combining, through the use of a maximal ratio combiner (MRC). We assume that the outputs of all the AEs in the service region are involved in the combining process rather than a subset. Such a system constitutes the logical limit of all practical macrodiversity implementations with linear combining.

There are basically two methods of realizing such a macrodiversity system. In the first approach, the received signals from a particular user would be demodulated at each AE and the corresponding decision variables would be relayed to the CS for combining. In this case, the complexity of an $\mathrm{AE}$ would almost be equal to that of a conventional base station. Alternatively, all the signal-specific processing can be centralized and performed at the CS. This would yield affordable and simple AEs, but would require wideband analog links between the AEs and CS [3]-[5]. The choice of realization does not affect the mathematical analysis; without loss of generality, we consider the second type of realization in this paper. 
Here are some further system-level assumptions.

- The service region is isolated in the sense that there is no interference coming from outside.

- The system is interference limited (thermal-type background noise is omitted).

- Only a single-class service is considered for the sake of simplicity, but the results and discussions can readily be extended to systems with multiclass services.

- An optimal power control scheme which yields SIR-balancing is employed [1], [6], [7]. Toward this end, it is assumed that: 1) all the link gains are known; 2) power levels are adjusted precisely and instantaneously; and 3) there are no constraints on the transmit power levels.

The distributed (limited-knowledge) implementation of SIRbalancing through iterative power control algorithms is demonstrated in the literature for conventional systems with single antennas [8]. The investigation of such distributed power control algorithms in multi-antenna systems constitutes an interesting research topic. ${ }^{4}$

\section{B. Antenna Gain Against Interference Versus Diversity Gain Against Fading}

The analytical treatment of macro- [11], [12] and microdiversity ${ }^{5}[13]-[15]$ schemes are different in the literature since these two diversity schemes are conventionally implemented in different ways. While coherent combining is possible in microdiversity schemes, selection (or switching) combining is used (mostly on a frame by frame basis) in macrodiversity types (e.g., the soft handoff scheme in IS-95 [16]), due to the distance between the AEs. ${ }^{6}$ However, since here we consider a macrodiversity scheme with instantaneous coherent combining, the analytical treatment for both cases would be similar.

In the macrodiversity system that we consider, at each branch of the combiner, in the receiver corresponding to a particular user at the CS, there will be a term due to the signal from the user of interest (this term will be referred to as the signal component) and an additional term due to the aggregate interference (background noise will be insignificant in the interference-limited systems).

When the number of AEs with independently fading signal components increase, the mass in the probability density function of the SIR at the output of the combiner concentrates more and more around the mean value. In the limit the probability density function becomes a delta function at its mean; this corresponds to the nonfading channel. This gain, as a result of the elimination of fades, is known as the diversity gain. In both macro- [16] and microdiversity [14], [18], most of this diversity gain is attained by using only a few AEs and the returns diminish quite rapidly by the addition of further AEs.

It is important to note that there is a potential for an additional gain when multiple AEs are utilized since more energy

\footnotetext{
${ }^{4}$ It can even intuitively be stated that the required power control dynamic range will be less in a multi-antenna system [9], [10], therefore, the implementation of such distributed power control algorithms is expected to be more feasible.

${ }^{5}$ Although microdiversity can be realized in various ways, in this paper it refers to the particular realization with multiple receiving AEs.

${ }^{6}$ Various suboptimal macrodiversity schemes which utilizes multiple AE have been suggested in the literature, such as the majority logic decoding [17].
}

is collected through multiple AEs; this results in an increased mean value for the SIR at the combiner output in addition to yielding a smoother SIR. This gain in the mean is simply due to building a more effective antenna and is known as the antenna gain. Conventionally, the antenna gain is not incorporated in the performance returns due to diversity combining (for instance, refer to [14, Fig. 14.4-2], in the context of microdiversity); it is understood that the antenna gain is in addition to the diversity gain [14, eq. (14.4-34)].

In a microdiversity system, in order to achieve diversity gain against multipath fading, the signal components at the combiner branches are not supposed to be significantly correlated. In a similar way, the extent of the antenna gain is inversely related to the severity of correlation between the interference or noise terms at the combiner branches, depending on whether the system is interference- or noise-limited, respectively. In a white noise-limited system, the noise terms at the combiner branches will be uncorrelated no matter how close the AEs are as long as they are not at the same point. It is this uncorrelatedness property that yields a mean output SNR (signal-to-noise ratio) in an MRC scheme which is the sum of the mean branch SNRs in white noise-limited systems [15], [18].

In this paper, we consider the reverse-link of an interference-limited finite-bandwidth CDMA macrodiversity system with maximal ratio combining. ${ }^{7}$ As it will be shown in the sequel, there is a possibility in this case that the interference terms at the combiner branches may turn out to be correlated if the AEs (or the interferers) are too close to one another. In such a case, the mean output SIR will be less than the sum of the mean branch SIRs. In the logical limit of identical interference terms, the combiner would reduce to that which has only one effective branch; therefore, there will not be any antenna gain at all, since amplification does not increase the SIR.

The notion of closeness of the AEs in the context of achieving antenna gain in a macrodiversity system is different than that in the context of achieving diversity gain in a microdiversity type. In a microdiversity system, the distance between the AEs is compared against the carrier wavelength-if the AEs are separated in the order of a few wavelengths, the level of correlation between the signal components at the combiner branches drops to insignificant levels and almost all of the diversity gain is attained [18], [21]. The major contribution of this paper is to introduce a similar parameter against which the distance between the AEs can be compared in interference-limited CDMA macrodiversity systems in the context of achieving antenna gain. It will be shown that the parameter which we will define as the chiplength ([speed of light]/[chip rate]) of the spreading code plays this role.

The scope of this paper is not the diversity gain (which is already well known), but the antenna gain that can be achieved in addition to the diversity gain, in the reverse-link of interference-limited CDMA macrodiversity systems. Therefore, we will analyze the correlation of the interference components at the combiner branches, not the correlation of the signal compo-

\footnotetext{
${ }^{7}$ We consider only the maximal ratio combining in this paper. It is important, however, to note that while the maximal ratio combining is the best combining technique in the presence of additive background noise, it is optimum combining that yields the best performance in the presence of interference [19], [20].
} 


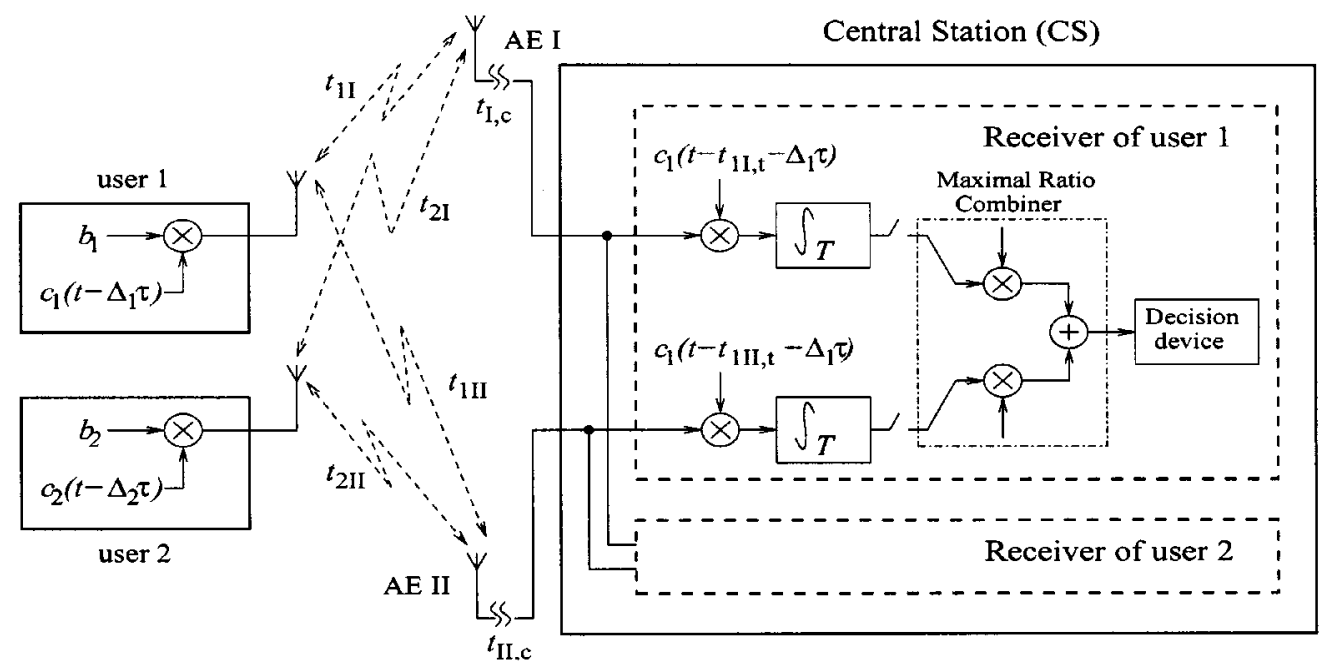

Fig. 1. The baseband transmitters and receivers with the propagation delays in the system.

nents. Since the nature of the antenna gain and diversity gain are different as explained in this section, we decouple the analyzes for these two types of gains and investigate here the antenna gain only. Toward that end, we consider a multi-AE system where the diversity gain against fading has already been obtained and thus, without loss of generality, where the fading is averaged out.

The rest of this paper is organized as follows. In Section II, the correlation coefficient analysis for the simplest nontrivial case of two AEs with two users is presented through the use of the concept of caution zones introduced in that section. In Section III, a way of approximating the caution zones is shown; and in Section IV, the effects of system parameters on the caution zones are discussed. The study of the spatial correlation effects is generalized to the case of many AEs with many users in Section $\mathrm{V}$ and the simulation results are presented in Section VI. A summary is given in Section VII with concluding remarks. Finally, the derivation of the correlation coefficient expression for Gold codes is provided in Appendix A and a systematic way of finding the approximate caution zones is explained in Appendix B.

\section{Correlation Coefficient AnAlysis For Two AEs WITH TWO USERS}

We consider a system with two AEs (AE $I$ and AE $I I$ ) and two wireless users $\left(w_{1}\right.$ and $\left.w_{2}\right)$. In the sequel, the indices $i$ and $j$ are used to denote a user and an AE, respectively, with $i \in\{1,2\}, j \in\{I, I I\}$. We assume that $w_{1}$ is the user of interest. The propagation delays in the system are illustrated in Fig. 1 along with the structure of the baseband transmitters and receivers. The total propagation time from $w_{i}$ to the CS through $\mathrm{AE} j, t_{i j, t}$, is equal to the sum of the propagation time in the air, $t_{i j}$ and that in the cable, $t_{j, c}: t_{i j, t}=t_{i j}+t_{j, c}$.

The spreading code for $w_{i}$ is represented as

$$
c_{i}(t)=\sum_{k=0}^{N-1} c_{i k} v(t-k \tau)
$$

where $N$ denotes the spread spectrum processing gain defined as the number of chips per bit (i.e., $N=T / \tau$ with $T$ and $\tau$ showing the bit and chip durations, respectively), $\left\{c_{i k}\right\}$ denotes the chip sequence with values equal to \pm 1 and $v(t)$ denotes the rectangular chip shaping function as follows: $v(t)=1$, if $0 \leq t<\tau$ and $v(t)=0$, otherwise. The cross-correlation between the spreading codes of $w_{1}$ and $w_{2}$, denoted by $R_{12}(q)$ where $q$ is an integer, is defined as

$$
R_{12}(q)=\left\langle c_{1}(t+q \tau) c_{2}(t)\right\rangle=\tau \sum_{k=0}^{N-1} c_{1, k+q} c_{2, k}
$$

where $\langle$.$\rangle shows the inner product, i.e., \left\langle c_{1}(t) c_{2}(t)\right\rangle=$ $\int_{T} c_{1}(t) c_{2}(t) d t$.

The baseband signal transmitted by $w_{i}$ (refer to Fig. 1) is $b_{i} c_{i}\left(t-\Delta_{i} \tau\right)$, where $b_{i}$ is the binary data with equiprobable values \pm 1 and $\Delta_{i}$ is the code phase for $w_{i}$ (asynchronous users). At $w_{1}$ 's receiver, the despreading at the $j$ th branch is performed by multiplying the received signal by $c_{1}\left(t-t_{1 j, t}-\Delta_{1} \tau\right)$.

We note that although $c_{1}(t) c_{2}(t) \neq c_{1}\left(t-t_{0}\right) c_{2}\left(t-t_{0}\right)$ (since the right-hand side is the time-shifted version of the left-hand side), the following equation holds: $\left\langle c_{1}(t) c_{2}(t)\right\rangle=$ $\left\langle c_{1}\left(t-t_{0}\right) c_{2}\left(t-t_{0}\right)\right\rangle$. Fig. 2 illustrates the difference between the product and inner product of two spreading codes; note that the former is a time function while the latter is a number. We also note that although

$$
\begin{aligned}
\left\langle c_{1}\left(t-t_{1}\right) c_{2}\left(t-t_{2}\right)\right\rangle & =\left\langle c_{1}\left(t-\left[t_{1}-t_{2}\right]\right) c_{2}(t)\right\rangle \\
& =\left\langle c_{1}(t) c_{2}\left(t-\left[t_{2}-t_{1}\right]\right)\right\rangle
\end{aligned}
$$

where $t_{0}, t_{1}$ and $t_{2}$ denote some arbitrary delays

$$
\begin{aligned}
\left\langle c_{1}\left(t-t_{1}\right) c_{2}(t)\right\rangle & \left\langle c_{1}\left(t-t_{2}\right) c_{2}(t)\right\rangle \\
& \neq\left\langle c_{1}\left(t-\left[t_{1}-t_{2}\right]\right) c_{2}(t)\right\rangle\left\langle c_{1}(t) c_{2}(t)\right\rangle
\end{aligned}
$$

for $t_{1} \neq t_{2}$; but rather, the left-hand side in (4) depends on the actual values of $t_{1}$ and $t_{2}$.

Now, let $\eta_{12 j}$ be the interference component (after despreading) at the branch of the MRC of $w_{1}$, due to $w_{2}$, corresponding to $\mathrm{AE} j$

$$
\eta_{12 j}=\left\langle c_{1}\left(t-t_{1 j, t}-\Delta_{1} \tau\right) b_{2} c_{2}\left(t-t_{2 j, t}-\Delta_{2} \tau\right)\right\rangle .
$$




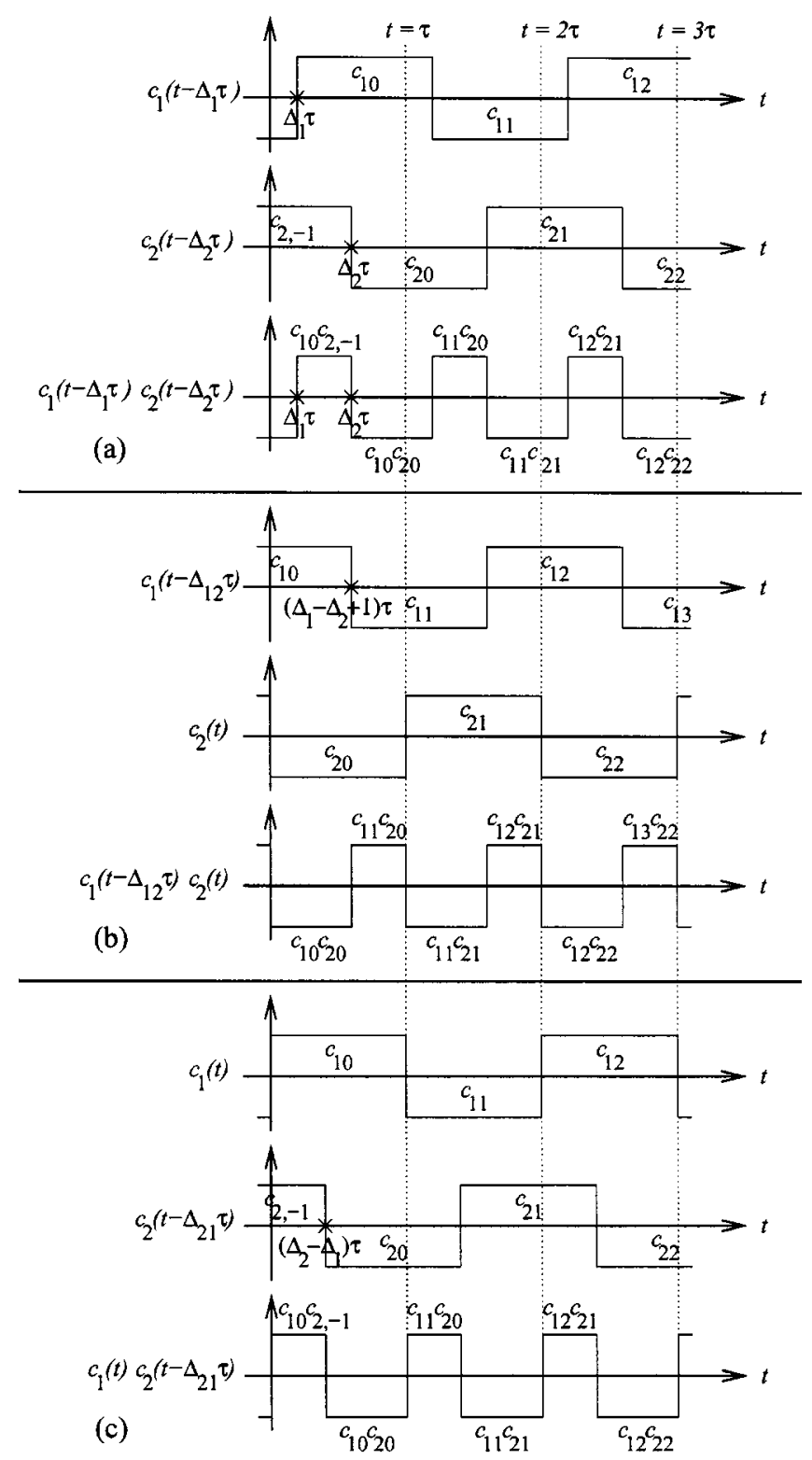

Fig. 2. Comparison of the product and inner product of two spreading codes: although (a) $c_{1}\left(t-\Delta_{1} \tau\right) c_{2}\left(t-\Delta_{2} \tau\right)$, (b) $c_{1}\left(t-\Delta_{12} \tau\right) c_{2}(t)$ and (c) $c_{1}(t) c_{2}\left(t-\Delta_{21} \tau\right)$, are all different time functions, $\left\langle c_{1}\left(t-\Delta_{1} \tau\right) c_{2}(t-\right.$ $\left.\left.\Delta_{2} \tau\right)\right\rangle=\left\langle c_{1}\left(t-\Delta_{12} \tau\right) c_{2}(t)\right\rangle=\left\langle c_{1}(t) c_{2}\left(t-\Delta_{21} \tau\right)\right\rangle$.

\section{A. Dependence of Correlation Coefficient on Propagation Delays}

As a starting point, we consider the case where the users are synchronized, in other words, $\Delta_{1}=\Delta_{2}$. The general case, where $\Delta_{1} \neq \Delta_{2}$ will be considered in Section II-C and it willbe apparent that the results obtained for synchronous users in this section can readily be generalized with minor modifications.

For the case of synchronous users, (3) can be used to simplify $\eta_{12 j}$, given by (5), as

$$
\eta_{12 j}=b_{2}\left\langle c_{1}\left(t-t_{12 j} \tau\right) c_{2}(t)\right\rangle
$$

where

$t_{12 j}=\frac{t_{1 j, t}-t_{2 j, t}}{\tau}=\frac{\left(t_{1 j}+t_{j, c}\right)-\left(t_{2 j}+t_{j, c}\right)}{\tau}=\frac{t_{1 j}-t_{2 j}}{\tau}$.

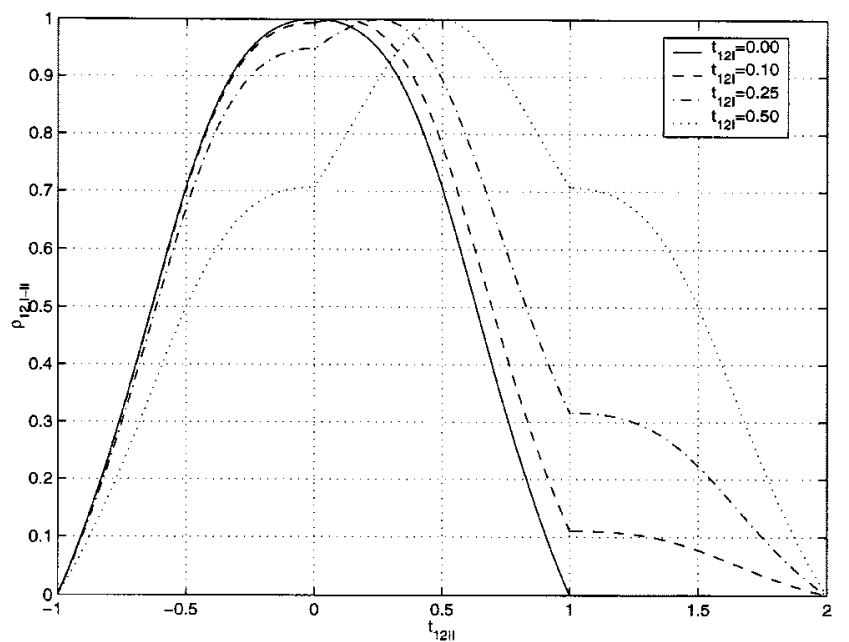

(a)

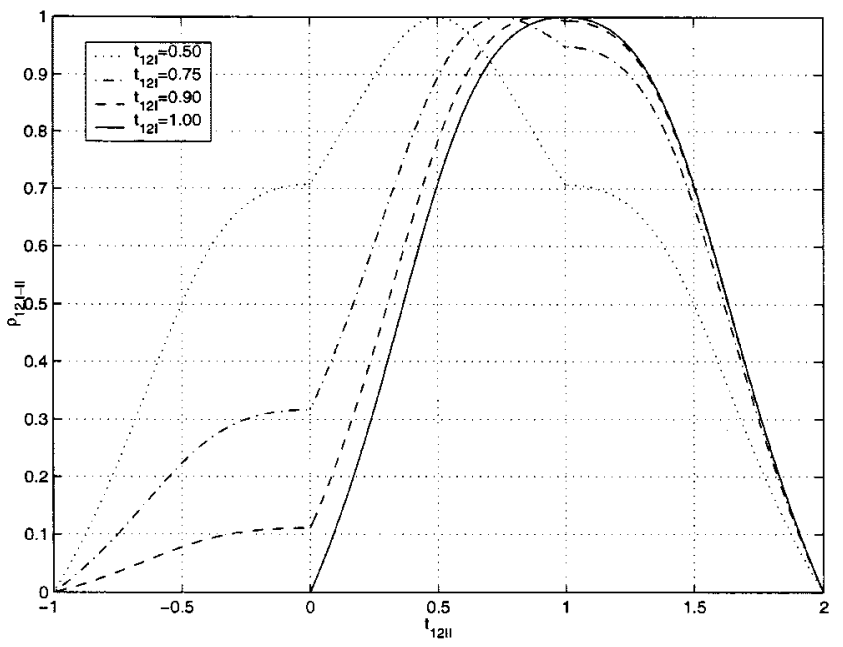

(b)

Fig. 3. Correlation coefficient as a function of $t_{12 I I}$, for $t_{12 I}=0.00,0.10$, $0.25,0.50,0.75,0.90$ and 1.00, for synchronous users (Bernoulli codes).

It is observed from (7) that $t_{12 j}$ depends only on the propagation delays in the air.

Let us first consider the case where $0 \leq t_{12 j} \leq 1$. For this region, $\eta_{12 j}$ can be evaluated from (1) and (6) as (refer to Fig. 2 as well)

$$
\eta_{12 j}=b_{2} \tau \sum_{k=0}^{N-1}\left[t_{12 j} c_{1, k-1} c_{2, k}+\left(1-t_{12 j}\right) c_{1, k} c_{2, k}\right]
$$

which can be rewritten using (2) as

$$
\eta_{12 j}=b_{2}\left[t_{12 j} R_{12}(-1)+\left(1-t_{12 j}\right) R_{12}(0)\right] .
$$

The $\eta_{12 j}$ expression given in (9) for $0 \leq t_{12 j} \leq 1$ can be generalized for any $t_{12 j}$ value as

$$
\begin{aligned}
\eta_{12 j}=b_{2}\left[\left(t_{12 j}-\left\lfloor t_{12 j}\right\rfloor\right)\right. & R_{12}\left(-\left\lceil t_{12 j}\right\rceil\right) \\
+ & \left.\left(\left\lceil t_{12 j}\right\rceil-t_{12 j}\right) R_{12}\left(-\left\lfloor t_{12 j}\right\rfloor\right)\right]
\end{aligned}
$$

where $\lfloor$.$\rfloor and \lceil$.$\rceil denote the floor and ceiling functions, respec-$ tively.

It is easy to show that $\mathbf{E}\left(\eta_{12 j}\right)=0$, where $\mathbf{E}($.$) denotes$ the expected value, since $b_{2}$ is zero-mean. We are interested in 
evaluating the correlation coefficient of the random variables $\eta_{12 I}$ and $\eta_{12 I I}, \rho_{12, I-I I}$, which is defined as

$$
\rho_{12, I-I I}=\frac{\mathbf{E}\left(\eta_{12 I} \eta_{12 I I}\right)}{\sqrt{\mathbf{E}\left(\eta_{12 I}^{2}\right)} \sqrt{\mathbf{E}\left(\eta_{12 I I}^{2}\right)}} .
$$

In this section ${ }^{8}$ we consider the case where $\left\{c_{i k}\right\}$ constitutes an equiprobable Bernoulli process ${ }^{9}$ with values equal to \pm 1 . In this case, $R_{12}(q)$ will be a random variable. It can be shown from (2) that

$$
\mathbf{E}\left(R_{12}(q)\right)=0, \forall q, \text { and } \mathbf{E}\left(R_{12}(q) R_{12}(r)\right)=N \tau^{2} \delta_{q-r}
$$

where $\delta$ is the Kronecker delta. Then, it follows from (10) and (12) that

$$
\mathbf{E}\left(\eta_{12 j}^{2}\right)=N \tau^{2}\left[\left(t_{12 j}-\left\lfloor t_{12 j}\right\rfloor\right)^{2}+\left(\left\lceil t_{12 j}\right\rceil-t_{12 j}\right)^{2}\right] .
$$

It can be observed from (8)-(10) and (12) that $\mathbf{E}\left(\eta_{12 I} \eta_{12 I I}\right)$ and thus $\rho_{12, I-I I}$, will be nonzero only when

$$
\left\lfloor t_{12 I}\right\rfloor-1 \leq t_{12 I I} \leq\left\lceil t_{12 I}\right\rceil+1
$$

which is equivalent to $\left\lfloor t_{12 I I}\right\rfloor-1 \leq t_{12 I} \leq\left\lceil t_{12 I I}\right\rceil+1$. Finally, from (11)-(13) $\rho_{12, I-I I}$ can be obtained as (15) at the bottom of the page.

For the sake of illustration, $\rho_{12, I-I I}$ is plotted in Fig. 3 for various values of $t_{12 I}$, in the range of $[0,1]$ and for $-1 \leq t_{12 I I} \leq 2$. In Fig. 5, the general expression for $\rho_{12, I-I I}$ given in (15) is plotted with respect to $t_{12 I}$ and $t_{12 I I}$. We note that in Fig. 5, the intersection of the vertical $\rho_{12, I-I I}-t_{12 I I}$ plane with the three-dimensional plot at certain values of $t_{12 I}$ yield the curves shown in Fig. 3.10

It is observed from (14), (15) and Fig. 5 that $t_{12 I}$ is shown as (16) at the bottom of the page. In (16), $m$ is an integer.

\section{B. Dependence of Correlation Coefficient on User and $A E$ Locations}

Our goal in this section is to find the region for $w_{2}$ which will result in a nonzero $\rho_{12, I-I I}$, for a given set of $\mathrm{AE}$ and

${ }^{8}$ Most of the intermediate steps in the development of the expressions in this section, especially those for (13) and (15), are omitted since they are straightforward. A more detailed discussion is presented in [22], but for a less general case with a different notation.

${ }^{9}$ The correlation coefficient analysis for Gold codes is presented in Appendix A.

${ }^{10}$ Similar curves for the case of Gold codes are shown in Fig. 4 (refer to Appendix A).

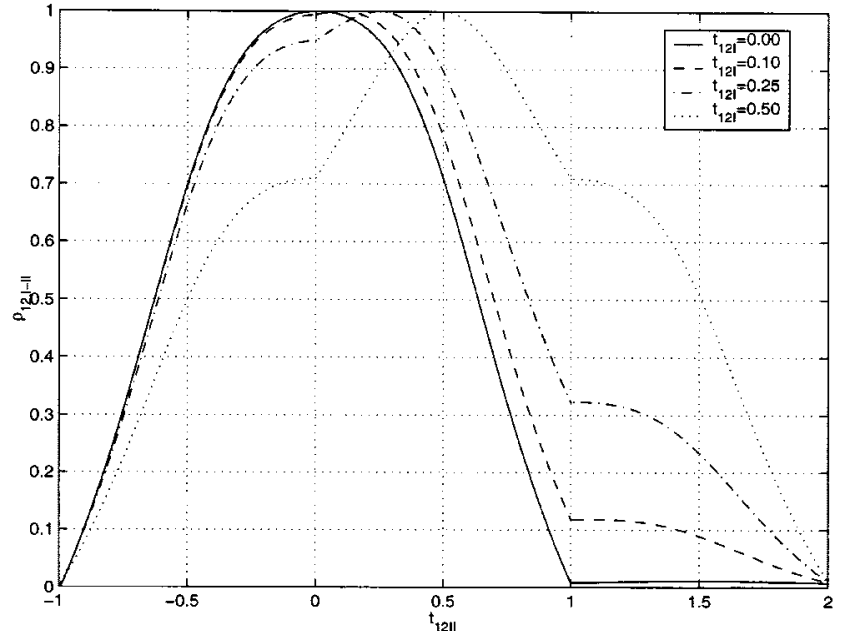

(a)

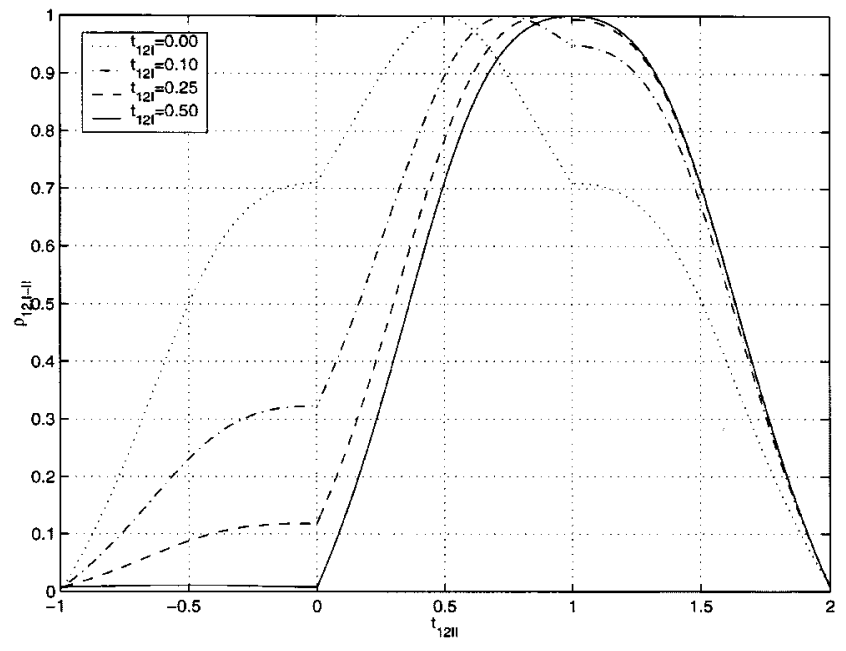

(b)

Fig. 4. Correlation coefficient as a function of $t_{12 I I}$, for $t_{12 I}=0.00,0.10$, $0.25,0.50,0.75,0.90$ and 1.00, for synchronous users (Gold codes).

$w_{1}$ locations; this region will be formally defined as the caution $z o n e$ for $w_{1}$ later in this section. The time domain requirements for this region is already known from (16). Therefore, the task is to find the equivalent of (16) in terms of distances involved.

As it is well known, the propagation time, $t$, depends on the distance, $d$, as $t=d / c$, where $c$ is the speed of light which will

$$
\begin{aligned}
\rho_{12, I-I I}=( & \left(\left\lceil t_{12 I}\right\rceil-t_{12 I}\right)\left(t_{12 I I}-\left\lfloor t_{12 I I}\right\rfloor\right) \delta_{\left\lfloor t_{12 I}\right\rfloor\left\lceil t_{12 I I}\right\rceil} \\
& +\left[\left(\left\lceil t_{12 I}\right\rceil-t_{12 I}\right)\left(\left\lceil t_{12 I I}\right\rceil-t_{12 I I}\right)+\left(t_{12 I}-\left\lfloor t_{12 I}\right\rfloor\right)\left(t_{12 I I}-\left\lfloor t_{12 I I}\right\rfloor\right)\right] \delta_{\left\lfloor t_{12 I}\right\rfloor\left\lfloor t_{12 I I}\right\rfloor} \\
& \left.+\left(t_{12 I}-\left\lfloor t_{12 I}\right\rfloor\right)\left(t_{12 I I}-\left\lfloor t_{12 I I}\right\rfloor\right) \delta_{\left\lceil t_{12 I}\right\rceil\left\lfloor t_{12 I I}\right\rfloor}\right) / \\
& \left(\sqrt{\left(t_{12 I}-\left\lfloor t_{12 I}\right\rfloor\right)^{2}+\left(\left\lceil t_{12 I}\right\rceil-t_{12 I}\right)^{2}} \sqrt{\left(t_{12 I I}-\left\lfloor t_{12 I I}\right\rfloor\right)^{2}+\left(\left\lceil t_{12 I I}\right\rceil-t_{12 I I}\right)^{2}}\right) .
\end{aligned}
$$

$$
\begin{gathered}
t_{12 I}=m \longrightarrow \begin{cases}\rho_{12, I-I I}=0, & \text { for } t_{12 I I} \leq m-1 \text { and } t_{12 I I} \geq m+1 \\
0<\rho_{12, I-I I} \leq 1, & \text { for } m-1<t_{12 I I}<m+1,\end{cases} \\
m<t_{12 I}<m+1 \\
\longrightarrow\left\{\begin{array}{ll}
\rho_{12, I-I I}=0, & \text { for } t_{12 I I} \leq m-1 \text { and } t_{12 I I} \geq m+2 \\
0<\rho_{12, I-I I} \leq 1, & \text { for } m-1<t_{12 I I}<m+2
\end{array} .\right.
\end{gathered}
$$




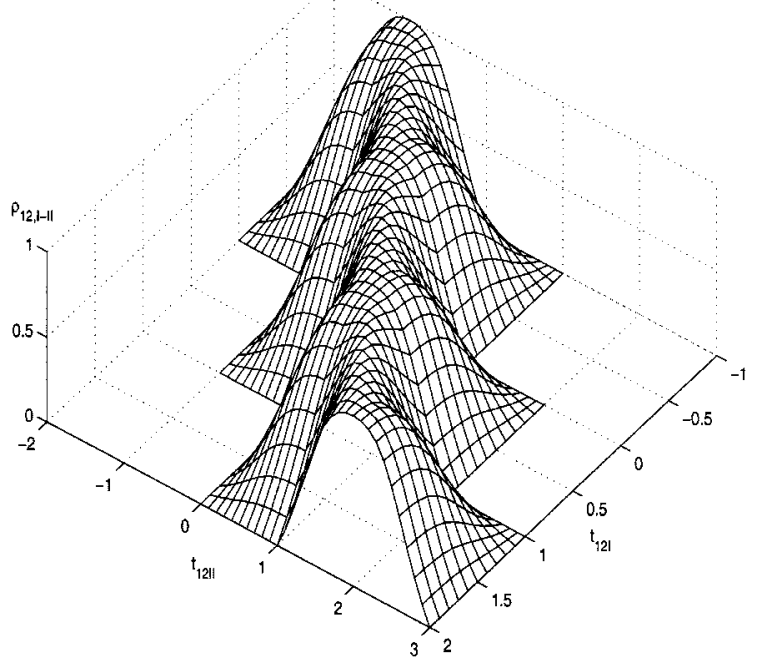

Fig. 5. Correlation coefficient as a function of $t_{12 I}$ and $t_{12 I I}$, for synchronous users.

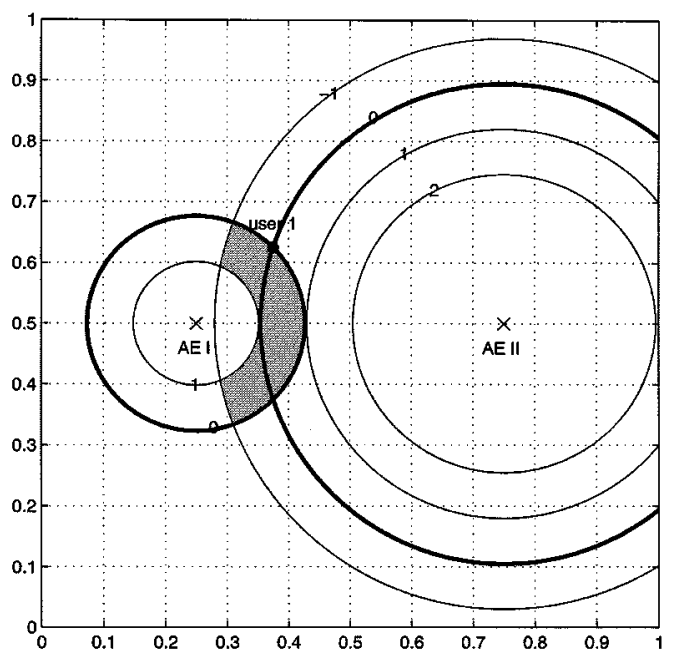

Fig. 6. For synchronous users, the shaded area shows the following region $0<\left(h_{1 I}-h_{2 I}\right)<0.075$ and $-0.075<\left(h_{1 I I}-h_{2 I I}\right)<0.15(s=400$ and $R_{c}=10 \mathrm{MHz}$ ).

be taken as $3 \times 10^{8} \mathrm{~m} / \mathrm{sec}$. Therefore, from (7), $t_{12 j}$ can be stated as

$$
t_{12 j}=\frac{d_{1 j}-d_{2 j}}{c \tau}
$$

where $d_{i j}$ is the Euclidean distance between $w_{i}$ and $\mathrm{AE} j$. There are two inherent assumptions in (17). First, it is assumed that the delay spread is much less in comparison to the propagation time in the air. Second, a direct path between each user and $\mathrm{AE}$ is implied due to the Euclidean distance assumption. In general, especially in urban environments where multi-antenna systems are more likely to be used, a user's signal is likely to arrive to an $\mathrm{AE}$ through a reflected path. In those cases, the caution zones will not have such regular shapes as will be shown in Figs. 6-14. However, since the actual propagation times will be greater or equal to those corresponding to direct paths, the actual caution zones will in general be smaller in real systems, which means that our performance results are conservative.

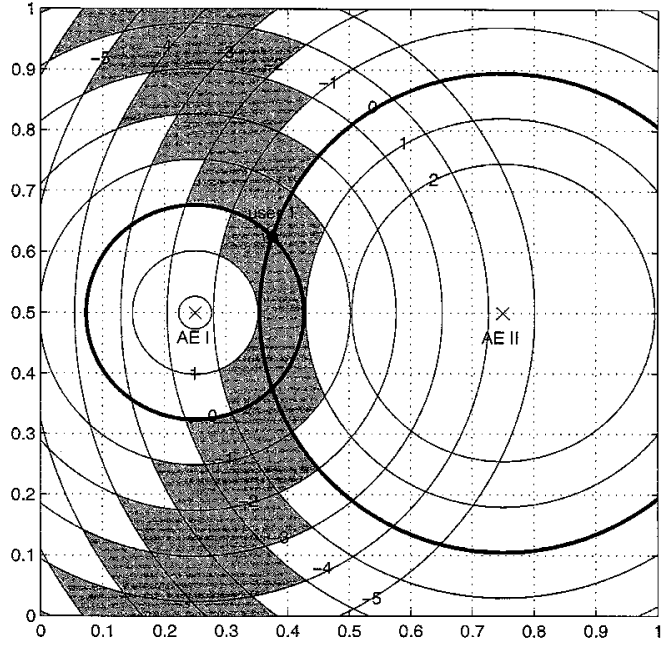

Fig. 7. The caution zone for $w_{1}\left(s=400\right.$ and $\left.R_{c}=10 \mathrm{MHz}\right)$, for synchronous users.

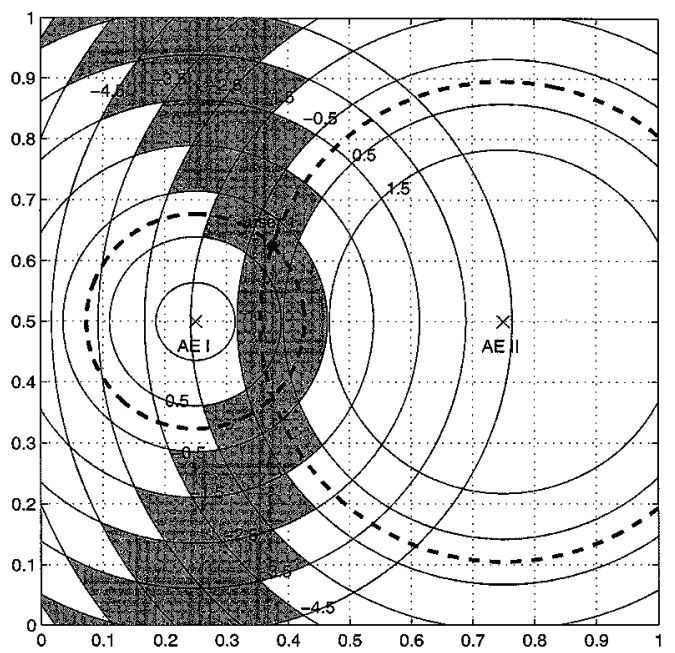

Fig. 8. The caution zone for $w_{1}\left(s=400\right.$ and $\left.R_{c}=10 \mathrm{MHz}\right)$, for asynchronous users with $\Delta_{12}=0.5$.

We will work on a unit square region with side length equal to 1 meter. Distances in this unit region will be denoted by $h$. In other words, $h$ is the normalized distance which is related to the actual distance, $d$, as

$$
d=h s
$$

where $s$ is the scaling factor. For instance, $s=500$ corresponds to a square region with side length $500 \mathrm{~m}$. Also, we define the chip rate, $R_{c}$, as $R_{c}=1 / \tau$.

We will first find the region corresponding to $m \leq t_{12 I} \leq$ $m+1$. This region is a function of the locations of $w_{1}$ and $w_{2}$ and $\mathrm{AE} I$ (but, not $\mathrm{AE} I I$ ). Then, we will find the region corresponding to $m-1 \leq t_{12 I I} \leq m+2$, which is a function of the locations of $w_{1}$ and $w_{2}$ and $\mathrm{AE} I I$ (but, not AE $I$ ).

For $m=0$, using (17) and (18), we can write

$$
\begin{aligned}
0 & \leq t_{12 I} \leq 1 \longrightarrow 0 \leq h_{1 I}-h_{2 I} \leq \frac{c \tau}{s} \\
-1 & \leq t_{12 I I} \leq 2 \longrightarrow-\frac{c \tau}{s} \leq h_{1 I I}-h_{2 I I} \leq 2 \frac{c \tau}{s} .
\end{aligned}
$$

It is observed from (16) that the intersection of these two regions yields the nonzero $\rho_{12, I-I I}$ region for $w_{2}$, for $m=0$. In a 


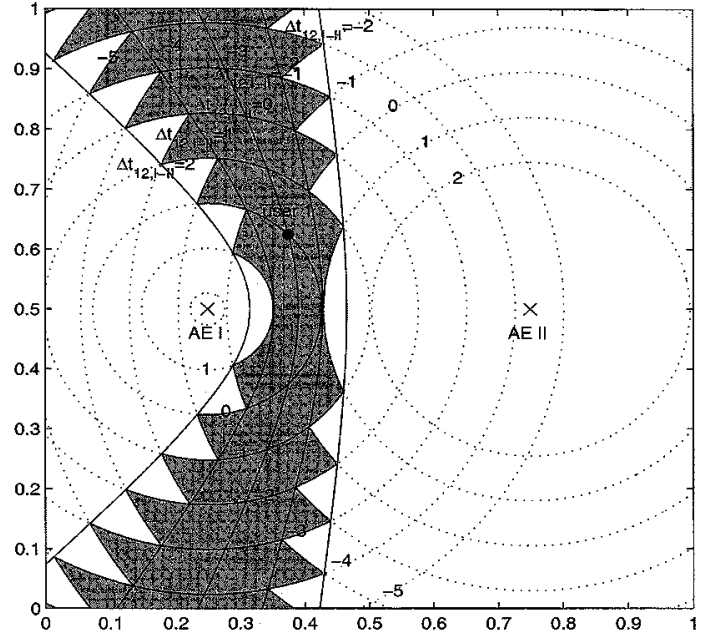

Fig. 9. The $\Delta t_{12, I-I I}=-2,-1,0,1$ and 2 lines along with the caution zone for $w_{1}$, for the case of synchronous users, with $s=400$ and $R_{c}=10 \mathrm{MHz}$.

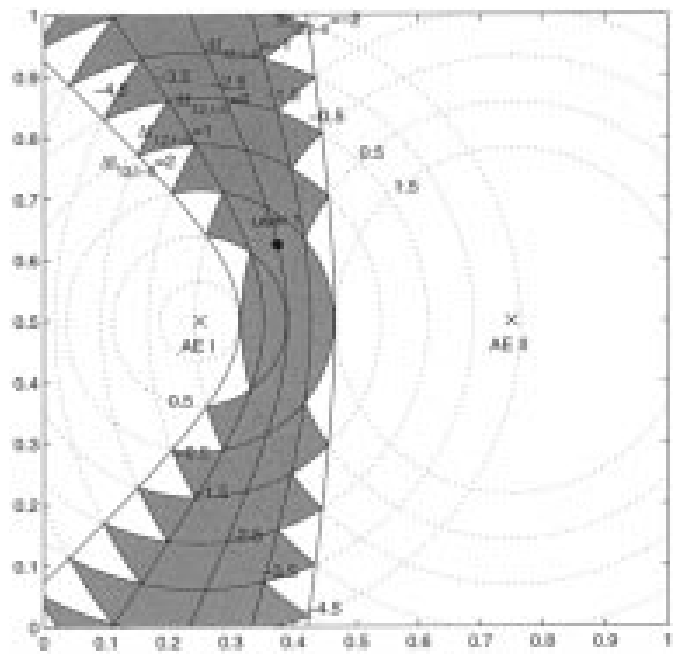

Fig. 10. The $\Delta t_{12, I-I I}=-2,-1,0,1$ and 2 lines along with the caution zone for $w_{1}$, for the case of asynchronous users $\left(\Delta_{12}=0.5\right)$, with $s=400$ and $R_{c}=10 \mathrm{MHz}$.

similar way, the corresponding regions for other $m$ values can also be found. We call the union of all such regions the caution $z o n e$ for $w_{1}$; because, the interference resulting from a user in this zone, at the $I$ th branch of $w_{1}$ 's combiner at the CS, will be correlated with the corresponding interference at the $I I$ nd branch.

As an example, we consider a system with $s=400$ and $R_{c}=10 \mathrm{MHz}$ (Megachips/sec), which results in $c \tau / s=0.075$. We assume that AEs $I, I I$ and $w_{1}$ are placed at the coordinates $(.25, .5),(.75, .5)$, and $(.375, .625)$, respectively, on the unit service region.

Fig. 6 shows the regions given by (19) and (20) as follows. In the figure, the numbers " 0 " and " 1 " on the left circles indicate that for any $w_{2}$ location on these circles, $t_{12 I}=0$ and 1 , respectively. The region for which $0 \leq\left(h_{1 I}-h_{2 I}\right) \leq 0.075$ would then be the area between these two circles. Similarly, the numbers " -1 " through " 2 " on the circles in Fig. 6 indicate that for any $w_{2}$ location on those circles, $t_{12 I I}=-1$, through 2 , respectively. Therefore, the region $-0.075 \leq\left(h_{1 I I}-h_{2 I I}\right) \leq 0.15$

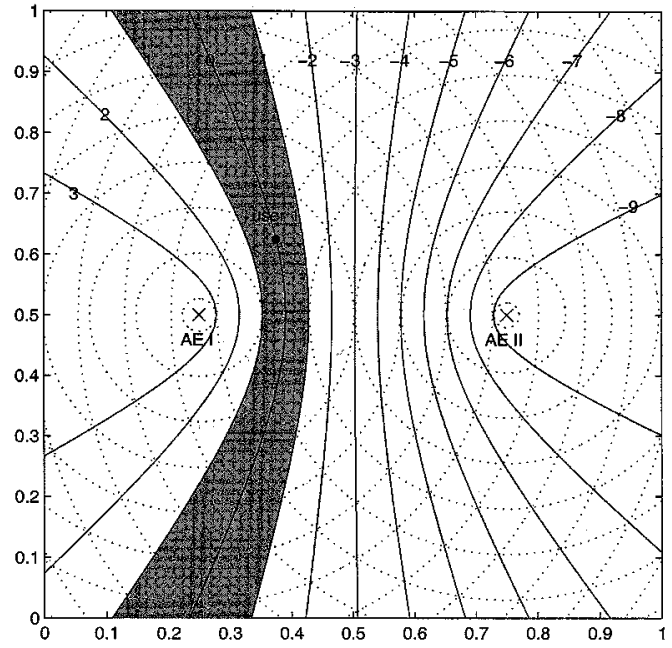

Fig. 11. The hyperbolic grid and approximate caution zone, for $s=400$, $R_{c}=10 \mathrm{MHz}$ and $w_{1}$ location $(.375, .625)$.

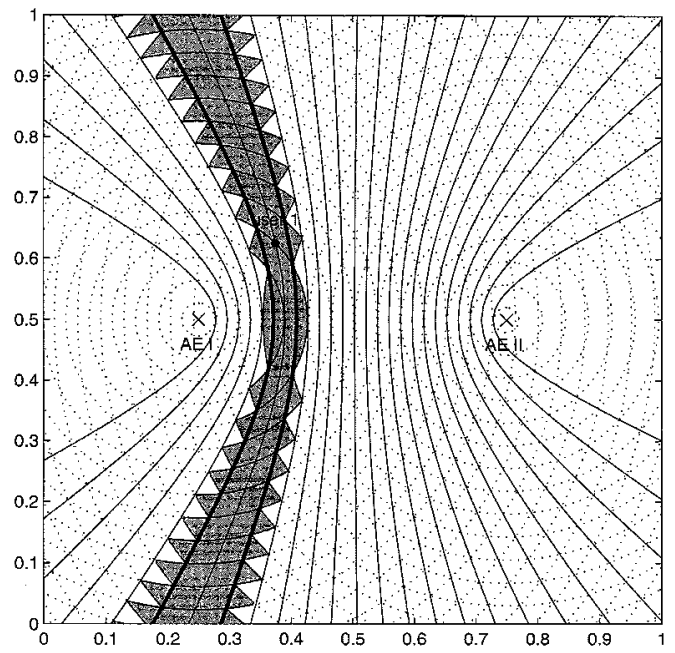

Fig. 12. The hyperbolic grid, actual and approximate caution zones, for $s=$ $800, R_{c}=10 \mathrm{MHz}$ and $w_{1}$ location $(.375, .625)$.

would now be the area between the two circles marks as -1 and 2 . The intersection of these two regions are shown by the shaded area in Fig. 6. Similarly, using (16)-(18), the corresponding regions for other $m$ values can also be obtained. We realize that for $m \geq 1$, the conditions given in (19) and (20) do not yield an overlapping region. In Fig. 7 the caution zone for $w_{1}$ is shown, which is the union of the regions corresponding to $m=-6,-5, \ldots, 0$.

\section{Correlation Coefficient Analysis for Asynchronous Users}

We note that the analysis presented so far is for the case where the users are synchronized. In this section, we turn our attention to the effect of the chip phase in the correlation analysis.

We define (normalized) differential code phase, $\Delta_{12}$, as the difference between the spreading code phases of $w_{1}$ and $w_{2}$; i.e., $\Delta_{12}=\Delta_{1}-\Delta_{2}$. Without loss of generality, $\Delta_{12}$ is modeled as a uniform random variable in the interval $[0,1)$. With this definition and based on (5)-(7), $\eta_{12 j}$ can be written as

$$
\eta_{12 j}=\left\langle c_{1}\left(t-t_{12 j} \tau-\Delta_{12} \tau\right) c_{2}(t)\right\rangle \text {. }
$$




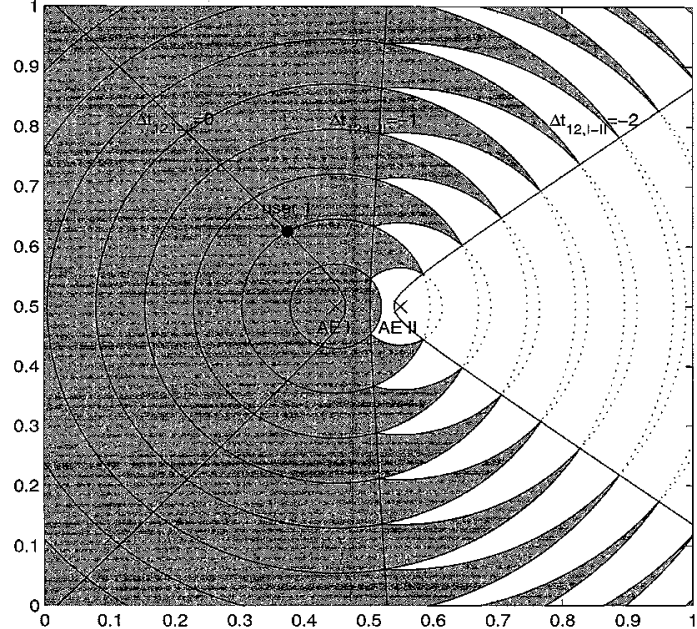

Fig. 13. The hyperbolic grid and approximate caution zone, for $s=400$ $R_{c}=10 \mathrm{MHz}, w_{1}$ location $(.375, .625)$ and $\mathrm{AE} I \& I I$ locations $(.45, .5)$ and $(.55, .5)$, respectively.

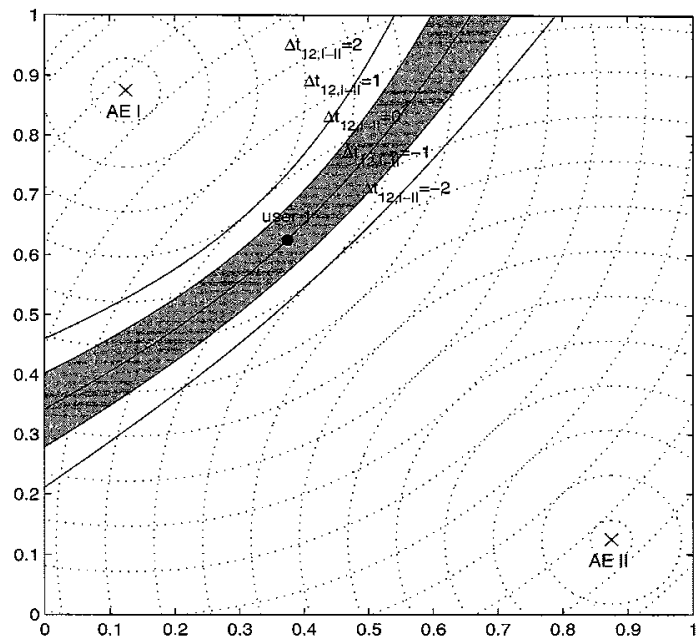

Fig. 14. The approximate caution zone, for $s=400, R_{c}=10 \mathrm{MHz}, w_{1}$ location $(.375, .625)$ and $\mathrm{AE} I \& I I$ locations $(.125, .875)$ and $(.875, .125)$, respectively.

Defining $t_{12 j}^{\prime}$ as

$$
t_{12 j}^{\prime}=t_{12 j}+\Delta_{12}
$$

(21) can be rewritten as

$$
\eta_{12 j}=\left\langle c_{1}\left(t-t_{12 j}^{\prime} \tau\right) c_{2}(t)\right\rangle .
$$

Comparing (23) with that for the synchronous users, namely, (6), we notice that both equations are in the same form with the only difference that $t_{12 j}$ in (6) is replaced with $t_{12 j}^{\prime}$ in (23). Therefore, we conclude that the $\rho_{12, I-I I}$ expressions found in Section II-A [such as (15)] as well as the curves plotted in Figs. 3

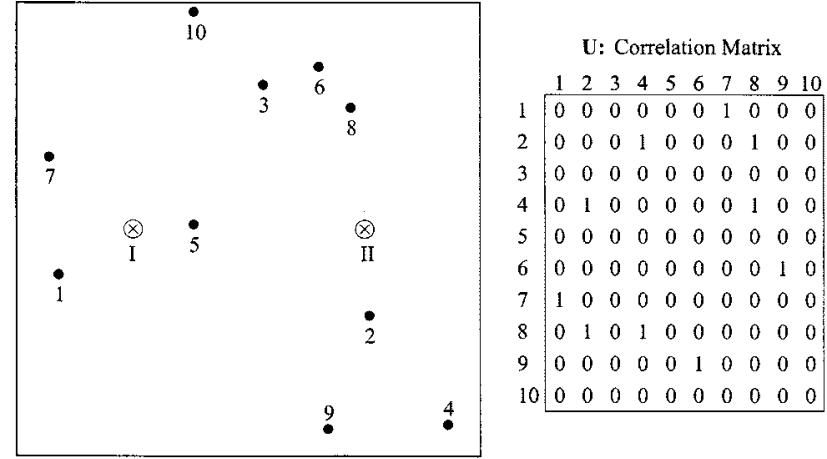

Fig. 15. A system with $L=2$ and $K=10$ and the corresponding correlation matrix, $\mathbf{U}$, for $s=400$ meters and $R_{c}=10 \mathrm{MHz}$.

and 5 are still valid provided that $t_{12 I}$ and $t_{12 I I}$ are substituted by $t_{12 I}^{\prime}$ and $t_{12 I I}^{\prime}$, respectively.

Based on (22) and the above observation, (16) can be modified as shown in (24) at the bottom of the page.

Following the same steps presented in Section II-B, the time domain region given in (24) for the nonzero $\rho_{12, I-I I}$ values can be converted into the expressions in terms of distances on the unit service region.

In Fig. 8, the caution zone for $w_{1}$ is shown for the case of $\Delta_{12}=0.5$. The shaded regions in Fig. 8 correspond to $m=$ $1,2, \ldots, 7$. Similar to Fig. 7 , the numbers " -4.5 " through " 0.5 " on the circles in Fig. 8, which have centers at $\mathrm{AE} I$, indicate that for any $w_{2}$ location on those circles, $t_{12 I}=-4.5$, through 0.5 , respectively. In the same way, the numbers " -4.5 " through " 1.5 ", on the circles which have centers at AE $I I$, indicate that for any $w_{2}$ location on those circles, $t_{12 I I}=-4.5$, through 1.5 , respectively.

\section{ApPRoXimation OF THE CAUTION Zones}

We start by defining the differential delay, $\Delta t_{12, I-I I}$, from $t_{12 I}^{\prime}$ and $t_{12 I I}^{\prime}$ (refer to (7) and (22)) as

$$
\begin{aligned}
\Delta t_{12, I-I I} & =t_{12 I}^{\prime}-t_{12 I I}^{\prime}=\left(t_{12 I}+\Delta_{12}\right)-\left(t_{12 I I}+\Delta_{12}\right) \\
& =\frac{\left(t_{1 I}-t_{2 I}\right)-\left(t_{1 I I}-t_{2 I I}\right)}{\tau}
\end{aligned}
$$

which can be rewritten as

$$
\Delta t_{12, I-I I}=\frac{\left(t_{1 I}-t_{1 I I}\right)-\left(t_{2 I}-t_{2 I I}\right)}{\tau} .
$$

We remark based on (25) and (26) that $\Delta t_{12, I-I I}$ is independent of the differential code phase, $\Delta_{12}$, that is, $\Delta t_{12, I-I I}$ is the same for both synchronous and asynchronous users.

We notice from (15), (16) and (24) that $\rho_{12, I-I I}$ depends on the actual values of $t_{12 I}$ and $t_{12 I I}$, rather than their difference, $\Delta t_{12, I-I I}$ (refer to (4)). In fact, it is observed from Fig. 5 that $\rho_{12, I-I I}$ exhibits periodicity with respect to $\Delta t_{12, I-I I}$ when $-2<\Delta t_{12, I-I I}<2$. The correlation analysis would have

$$
\begin{gathered}
t_{12 I}=m-\Delta_{12} \longrightarrow\left\{\begin{array}{l}
\rho_{12, I-I I}=0, \quad \text { for } t_{12 I I} \leq(m-1)-\Delta_{12} \text { and } t_{12 I I} \geq(m+1)-\Delta_{12} \\
0<\rho_{12, I-I I} \leq 1, \quad \text { for }(m-1)-\Delta_{12}<t_{12 I}<(m+1)-\Delta_{12},
\end{array}\right. \\
m-\Delta_{12}<t_{12 I}<(m+1)-\Delta_{12} \longrightarrow \begin{cases}\rho_{12, I-I I}=0, & \text { for } t_{12 I I} \leq(m-1)-\Delta_{12} \text { and } t_{12 I I} \geq(m+2)-\Delta_{12} \\
0<\rho_{12, I-I I} \leq 1, & \text { for }(m-1)-\Delta_{12}<t_{12 I I}<(m+2)-\Delta_{12} .\end{cases}
\end{gathered}
$$




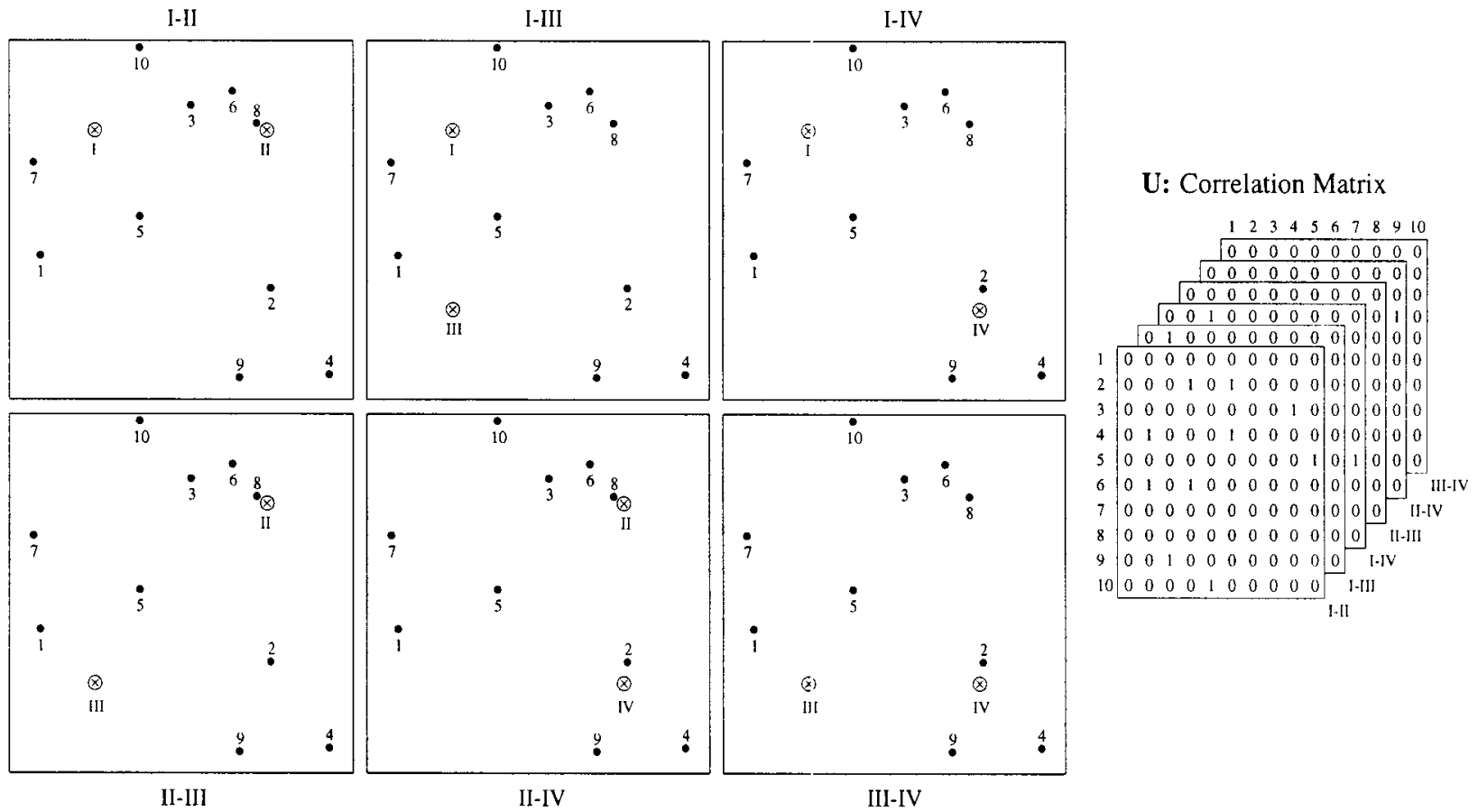

Fig. 16. A system with $L=4$ and $K=10$ and the corresponding correlation matrix, $\mathbf{U}$, for $s=400 \mathrm{~m}$ and $R_{c}=10 \mathrm{MHz}$.

been much simpler if $\rho_{12, I-I I}$ were constant (rather than being periodic) with respect to $\Delta t_{12, I-I I}$. In that case, a two-dimensional plot of $\rho_{12, I-I I}$, as a function of $\Delta t_{12, I-I I}$ would have been sufficient.

It is clear from Figs. 3 and 5 that $t_{12 I}=t_{12 I I}$ corresponds to the worst case in the correlated interference analysis and that $\left|t_{12 I}-t_{12 I I}\right|>2$ guarantees uncorrelatedness. In other words, it can be stated using (25) that

$$
\begin{aligned}
& \left|\Delta t_{12, I-I I}\right|=0 \longrightarrow \rho_{12, I-I I}=1 \\
& \left|\Delta t_{12, I-I I}\right| \geq 2 \longrightarrow \rho_{12, I-I I}=0 .
\end{aligned}
$$

One other important observation from Fig. 3 is that

$$
1 \leq\left|\Delta t_{12, I-I I}\right|<2 \longrightarrow 0 \leq \rho_{12, I-I I} \leq 0.5 \text {. }
$$

Obviously, it is desirable to have $\rho_{12, I-I I}=0$. However, it is important to note that even in the case of a $\rho_{12, I-I I}$ very close to unity, there will still be some gain against interference from combining, although this gain will be minuscule. In a practical system, however, if $\rho_{12, I-I I}$ is close to unity, the insignificant returns in mean SIR will not justify the increased processing, complexity and thus, cost.

It would be efficient if a threshold value for $\rho_{12, I-I I}$ is determined, say $\rho_{o}$, such that it can be argued that if $\rho_{\circ}<\rho_{12, I-I I} \leq$ 1 , then practically there would not be much antenna gain. We note that in order for the mean output SIR in a MRC scheme to be the sum of the mean branch SIRs, $\rho_{12, I-I I}$ should be equal to 0 . Therefore, for $\rho_{12, I-I I}=\rho_{o}$, as described above, the mean output SIR will be less than the sum of the mean branch SIRs. We choose $\rho_{O}=0.5$. Based to (27) and (29), setting $\rho_{O}$ to 0.5 is equivalent to the following inequality:

$$
\left|\Delta t_{12, I-I I}\right|>1 \text {. }
$$

The implication of (30) is that the caution zones (the shaded areas) in Figs. 9 and 10 can be approximated by the area between the $\Delta t_{12, I-I I}=-1$ and 1 lines. We know from (29) that $\rho_{12, I-I I}$ is less than 0.5 in the shaded regions outside the approximated caution zones, therefore, the performance degradation introduced by such an approximation is not expected to be significant.

Note that $\Delta t_{12, I-I I}=-1$ and 1 lines are hyperbolas. If we draw all the hyperbolas for which $\Delta t_{12, I-I I}$ is an integer, then we construct a hyperbolic grid. The "origin" of the hyperbolic grid is the hyperbola on which $w_{1}$ is located. A systematic way of finding the approximate caution zones on the unit service region is presented in Appendix B (see Fig. 11).

To the best of our knowledge, the only work in the literature on space-related interference correlation analysis in CDMA widely-spaced multi-antenna systems is presented in [1, Sect. 7] which is rather brief and qualitative.

It is stated in [1] that the correlation will depend on $\left|\Delta t_{12, I-I I}\right|<1$. We have shown in this paper, however, that $\left|\Delta t_{12, I-I I}\right|<1$ corresponds only to an approximation of the areas where the correlated interference exists. The actual such areas (caution zones) depend on the values of $t_{12 I}$ and $t_{12 I I}$ (refer to, for example, Figs. 3 and 5).

Also, in [1], a small area around a user is considered as the caution zone (in our terminology). However, we have demonstrated analytically and shown in numerous figures that the caution zones are, in fact, much broader due to the geometry of the problem. Two users that are at different sides of the service region may cause significant correlated interference to one another.

\section{A NeW System PARAMETER: ChiPlength}

It is obvious from the discussion so far that the existence and severity of the correlated interference depend on the value of $\left|\Delta t_{12, I-I I}\right|$. It is observed from (44) that $\left|\Delta t_{12, I-I I}\right|$, in turn, depends on two factors: the term $s R_{c} / c$ and the relative positions of the users and AEs (which are captured through $h_{i j}$ 's). 
It is noticed that for a certain value of $s R_{c} / c$, the actual values of $s$ and $R_{c}$ do not matter. Hence, as long as the correlated interference analysis is concerned, a system with $s=400$ and $R_{c}=10 \mathrm{MHz}$, for instance, is equivalent to that with $s=1000$ and $R_{c}=4 \mathrm{MHz}$.

It is well known that in microdiversity systems, in order to attain a gain against fading, the inter-AE distance should be at least a few times greater than the wavelength of the carrier, $\lambda=c / f$, where $f$ denotes the carrier frequency [18], [21]. In a CDMA macrodiversity system, a similar quantity which we will refer to as the "chiplength" of the spreading code can be defined as follows:

$$
\Lambda=\frac{c}{R_{c}} \mathrm{~m} .
$$

It will become apparent through the simulation results presented in Section VI that the role $\Lambda$ plays in macrodiversity systems (in regards to gain against interference) is indeed similar to that $\lambda$ plays in microdiversity types (in regards to gain against fading).

With (31), $s R_{c} / c$ reduces to $s / \Lambda$. For a large $s / \Lambda$ value, the hyperbolic grid will be denser; in other words, the hyperbolas, for which $\left(\vartheta_{1}-\vartheta_{2}\right) s / \Lambda$ is an integer (see Appendix B), will be closer to each other. Since the approximate caution zone is the area between the hyperbolas -1 and 1 , this area will be smaller Therefore, it is desirable to have a large $s / \Lambda$ value. In Fig. 12, the hyperbolic grid, the actual and approximate (thick lines) caution zones for $w_{1}$ are shown for the case of $s / \Lambda=26.67$, with $w_{1}$ location $(.375, .625)$. This may, for instance, correspond to a system with $s=800$ and $R_{c}=10 \mathrm{MHz}$. We note that in the example given in Section II-B, $s / \Lambda=13.33$ (see Fig. 11).

The density and orientation of the hyperbolic grid also depend on the AE locations in the service area. For a given $s / \Lambda$ value, the density of the hyperbolic grid will increase with the increasing distance between the AEs. Therefore, to minimize the caution zone (and thus, the effects of the correlated interference), AEs must be placed as far apart as possible ${ }^{11}$ —an intuitively satisfying result.

We will demonstrate this result by two examples. Fig. 13 shows the hyperbolic grid and the actual caution zone for a system where the two AEs are in very close proximity. In this case, the approximate caution zone covers almost half of the service area! Fig. 14, on the other hand, shows the hyperbolic grid and the approximate caution zone for a system where the two AEs are far apart. As expected, the corresponding approximate caution zone is much smaller. Note that for both of the systems illustrated in Figs. 13 and 14, $s / \Lambda$ value $(s=400$ and $\left.R_{c}=10 \mathrm{MHz}\right)$ and the $w_{1}$ location $(.375, .625)$ are the same.

In the rest of this paper, the term caution zone will be used to indicate the approximate caution zone (that is, the area between the hyperbolas $\Delta t_{12, I-I I}=-1$ and 1), unless otherwise stated.

These results are in agreement with those presented in [1]: in the limiting case of infinite bandwidth $\left(R_{c} \rightarrow \infty\right.$, thus $\left.\Lambda \rightarrow 0\right)$ the caution zone reduces to a line and the probability of $w_{2}$ being on this line approaches zero.

\footnotetext{
${ }^{11}$ This is true for an isolated system. If, however, there are adjacent or neighboring systems, the effect of inter-system interference on the AE locations should be taken into account.
}

\section{Percent Correlation Analysis}

In this section, we further the investigation of the spatial correlation analysis by considering, first, two AEs with many users and then, the most general case of many AEs with many users. Due to the computational complexity, however, we work with an intermediate performance metric, which we call percent correlation, instead of the correlation coefficient itself.

\section{A. Two AEs With Many Users}

In a system with $K$ users, for each user, we determine whether the remaining $K-1$ users are in the caution zone for that particular user. By this way, we construct a $K \times K$ correlation matrix, $\mathbf{U}=\left\{u_{k l}\right\}$, such that

$$
u_{k l}= \begin{cases}1, & \text { if } w_{k} \text { is in the caution zone for } w_{l} \\ 0, & \text { otherwise. }\end{cases}
$$

We note that $u_{k \cdot k}=0, k=1,2, \ldots, K$, since a user does not create interference to itself. Also, it can be shown that if $w_{l}$ is in the caution zone for $w_{k}$, then $w_{k}$ must be in the caution zone for $w_{l}$. It can further be shown that if $w_{l}$ is in the caution zone for $w_{k}$ and $w_{m}$ is in the caution zone for $w_{l}$, then this does not necessarily mean that $w_{m}$ will be in the caution zone for $w_{k}$. Therefore, $\mathbf{U}$ is a symmetric nontransitive matrix.

In Fig. 15, a system with $L=2$ and $K=10$ is illustrated and the corresponding $\mathrm{U}$ matrix is given $\left(s=400 \mathrm{~m}\right.$ and $R_{c}=$ $10 \mathrm{MHz}$ ). In Fig. 15, the caution zones are not drawn. But, if we were to draw the caution zone for $w_{8}$, for instance, then $w_{2}$ and $w_{4}$ would be in that caution zone. Consequently, $u_{82}=1$, $u_{84}=1$ and all the other entries in the 8th row of the $\mathbf{U}$ matrix are 0's.

In the worst case, all of the $K-1$ entries in a row of will be 1 's and in the best case, all of those entries will be 0 's. Based on this observation, we define the percent correlation for $w_{k}, \phi_{k}$, as follows:

$$
\phi_{k}=\frac{1}{K-1} \sum_{l=1}^{K} u_{k l} \times 100, k=1,2, \ldots, K .
$$

It is worth noting that although $\phi$ is not equal to the correlation coefficient, there is a direct relation between them; $\phi=0 \%$ and $100 \%$ correspond to (but not necessarily identical to) $\rho=$ 0 and 1 , respectively.

\section{B. Many AEs With Many Users}

Obviously, the most interesting case is the most general type of $L$ AEs with $K$ users. We assume that the AEs are evenly placed on the service region, so that the coordinates of the $j$ th $\mathrm{AE}$ on the unit square region can be represented by the pair

$$
\left(\frac{2[(j-1) \bmod \sqrt{L}]+1}{2 \sqrt{L}}, 1-\frac{2\left\lceil\frac{j}{\sqrt{L}}\right\rceil-1}{2 \sqrt{L}}\right) .
$$

Now, for each user, a total of $\left(\begin{array}{l}L \\ 2\end{array}\right)=L(L-1) / 2$ caution zones exist, each of which corresponds to a particular AE pair. Therefore, the $\mathbf{U}$ matrix is composed of $L(L-1) / 2$ submatrices (one for each antenna pair), with sizes $K \times K$. Consequently, 
the correlation matrix, $\mathbf{U}=\left\{u_{k l r}\right\}$, is three -dimensional with size $K \times K \times[L(L-1) / 2]$, and

$$
u_{k l r}= \begin{cases}1, & \text { if } w_{l} \text { is in the caution zone for } w_{k} \\ & \text { with respect to the } r \text { th antenna pair } \\ 0, & \text { otherwise. }\end{cases}
$$

Such a system, for the case of $L=4$ and $K=10$, is illustrated in Fig. 16.

For a $w_{k}$, the most disadvantageous situation will occur if all of the remaining $K-1$ users are in all of the $L(L-1) / 2$ caution zones for $w_{k}$. Obviously, this is an event with a very low likelihood! Such a situation will yield $(K-1)[L(L-1) / 2] 1$ 's in the two-dimensional $k$ th row of the $\mathbf{U}$ matrix. Based on this observation, $\phi_{k}$ can be stated as

$$
\begin{aligned}
\phi_{k} & =\frac{1}{(K-1) \frac{L(L-1)}{2}} \sum_{r=1}^{L(L-1) / 2} \sum_{l=1}^{K} u_{k l r} \times 100, \\
k & =1,2, \ldots, K .
\end{aligned}
$$

We note that (36) reduces to (33) for $L=2$.

\section{SimUlation RESULtS}

Simulations have been run to obtain the $\phi$ values for various combinations of the system parameters with the assumption of uniform user distribution. For each such combination, a total of approximately $40000 \phi$ values are collected and the corresponding cumulative distribution function is plotted.

It is worth noting that if the number of users are identical in two systems which are compared, then the users in the system with higher (lower) $\phi$ values will experience a lower (higher) balanced mean SIR value. In order to compensate for (exploit) this effect, the number of users in that system has to be reduced (can be increased).

Before presenting the simulation results, we would like to make the following remarks for the proper interpretation. An increase in antenna gain by a certain factor will yield an increase in the mean SIR level by the very same factor, which in turn, will yield a capacity increase almost by that factor as well. The relation between the number of AEs and the antenna gain, on the other hand, depends on the severity of correlated interference-an increase by the same factor will happen only when the spreading bandwidth is infinite as stated throughout this paper. Therefore, a capacity penalty will be incurred due to the presence of the correlated interference effects when the bandwidth is finite. The simulation results given in this section do not quantify this penalty; but, they rather show the degree of interference, in terms of $\phi$, with respect to the key system parameters (namely, the spread spectrum bandwidth [chip rate] and the inter-AE distance which itself depends on the service region size). Obviously, a high $\phi$ value implies a greater departure from the linear capacity gain. In the limiting case of $\phi=100 \%$, there will be almost no antenna gain from using multiple AEs instead of a single AE.

\section{A. Percent Correlation and (Service Region Size)/(Chiplength)}

In Fig. 17, the relation between $s$ and $\Lambda$ is shown by fixing the number of AEs to 4 . Each of the three sets of curves in this figure, labeled as I, II and III, corresponds to a different
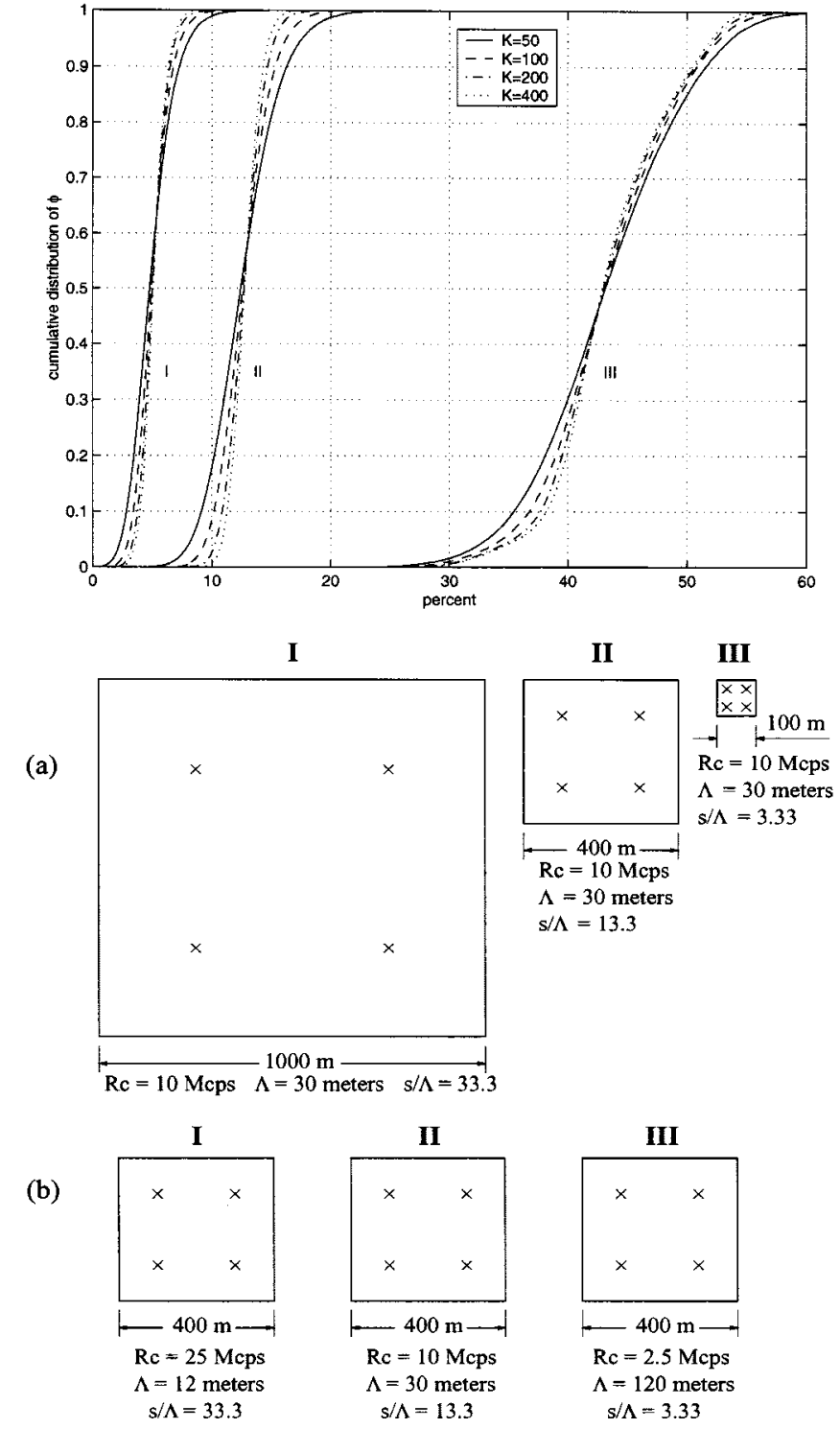

Fig. 17. Cumulative distribution function of $\phi$ for various $s / \Lambda$ values: interpretation (a) chip rate is kept constant and service region size is changed; interpretation (b) service region size is kept constant and chip rate is changed.

$s / \Lambda$ ratio: $33.3,13.3$ and 3.3, respectively. Fig. 17 (a) and (b) provide two different interpretations for the above given $s / \Lambda$ values. In Fig. 17(a), $\Lambda$ is kept constant at 30 meters (that is, $R_{c}=10 \mathrm{MHz}$ ) and the size of the service region is changed by reducing $s$ from 1000 to 400 to 100 . Note that since the AEs are uniformly placed, decreasing the service region size results in decreasing the inter-AE distance as well. In Fig. 17(b), on the other hand, the size of the service region is kept constant $(s=400)$ and $\Lambda$ is increased from 12 to 30 to $120 \mathrm{~m}$ (that is, $R_{c}$ is decreased from 25 to 10 to $2.5 \mathrm{MHz}$ ).

In order to investigate Fig. 17 closely, let us assume that the number of users is fixed ( $K=50,100,200$, or 400 ). The curves in Fig. 17 confirm our expectation that for a given number of AEs, decreasing $s / \Lambda$ (i.e., decreasing the service region size while keeping the spread spectrum bandwidth fixed, or decreasing the spread spectrum bandwidth while keeping the size of the service region fixed) yields greater correlation between the interference components received at different 

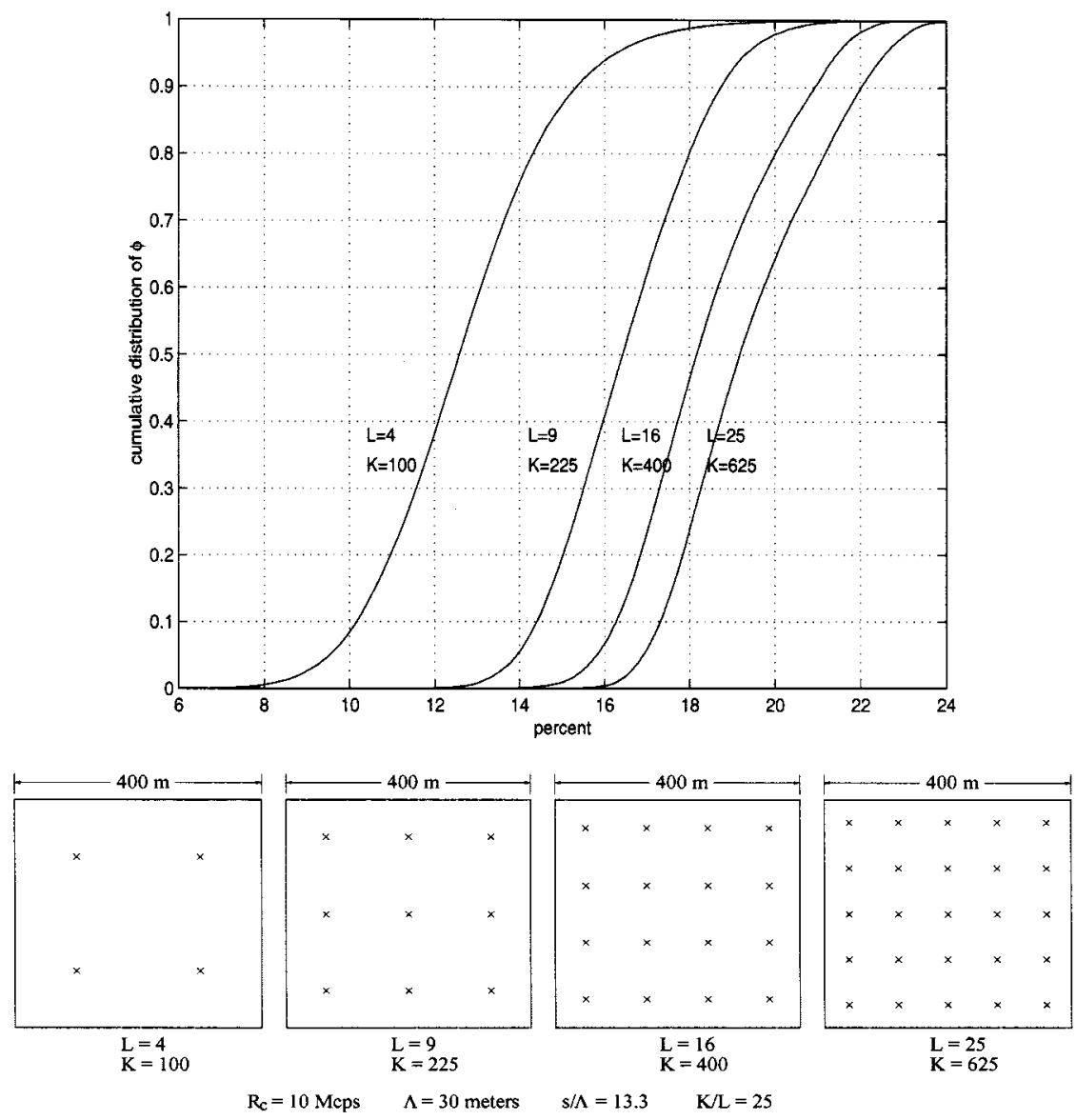

Fig. 18. Cumulative distribution function of $\phi$ for various inter-AE distances.

AEs. Furthermore, the increase in correlation is relatively more significant when $s / \Lambda$ is reduced from 13 to 3.3 , in comparison to when it is reduced from 33 to 13 . This suggests a nonlinear relationship between $s / \Lambda$ and $\phi$.

Let us reiterate one main point by considering scenarios I and III depicted in Fig. 17(b) and by comparing them with scenarios having only single $\mathrm{AE}$ while keeping all other parameters unchanged. When $L$ is increased from 1 to 4 , there will be some diversity gain and in addition to that, some antenna gain which is the topic of this paper. The cumulative distribution functions for $\phi$ shown in Fig. 17 suggest that the antenna gain will be close to 4 in scenario I $\left(R_{c}=25 \mathrm{MHz}\right)$, but that gain will be more modest in scenario II $\left(R_{c}=2.5 \mathrm{MHz}\right)$. However, as it was stated previously, the curves do not reveal the actual values of the antenna gains in those scenarios.

One other observation from Fig. 17 is the following: for a given $s / \Lambda$ value, the effect of the number of users on $\phi$ is marginal in all three cases (I, II, and III). ${ }^{12}$ This is due to the fact that the user locations are taken to be two-dimensional uniform random variables. Statistically, the percentage of users that are in a caution zone are determined by the size of that caution zone (in percentage) in comparison to the total size of the service region. Therefore, increasing the number of users reduces the sta-

\footnotetext{
${ }^{12}$ Obviously, as $K$ increases (decreases) while the other parameters remain unchanged, the balanced SIR value that users will experience will decrease (increase) accordingly.
}

tistical variations and as $K$ increases the tails of the distributions become less significant. However, the median values remain almost the same in each set, as expected.

\section{B. Percent Correlation and Inter-AE Distance}

The change in $\phi$ with respect to the number of AEs is investigated in Fig. 18 for a fixed $s / \Lambda$ value of 13.3. In a fixed service region, increasing $L$ means decreasing the inter-AE distance. In this figure, the ratio of the number of users to AEs is also kept constant $(K / L=25) .{ }^{13}$ It is observed, as expected, that $\phi$ increases with the increasing $L$; however this increase in percent correlation is quite mild. For instance, the increase in the median values of $\phi$ for the $L=25$ and $K=625$ case, in comparison to the $L=16$ and $K=400$ and to the $L=4$ and $K=100$ cases, are less than $1 \%$ and around $7 \%$, respectively. This would mean that the output SIR for a system with $L=25$ and $K=625$ will only be slightly lower than that with $L=4$ and $K=100$.

The conclusion from this figure is that in systems with relatively high $s / \Lambda$ values, even though the increase in antenna gain with the increasing number of AEs will be less than linear, this increase will not saturate rapidly. Therefore, there is room for significant capacity gains with the use of multiple AEs in such systems.

\footnotetext{
${ }^{13}$ We note based on Fig. 17 that if $K$ is varied for a given $L$ value, the tails of the distribution will be affected, but the median value will not change noticeably.
} 

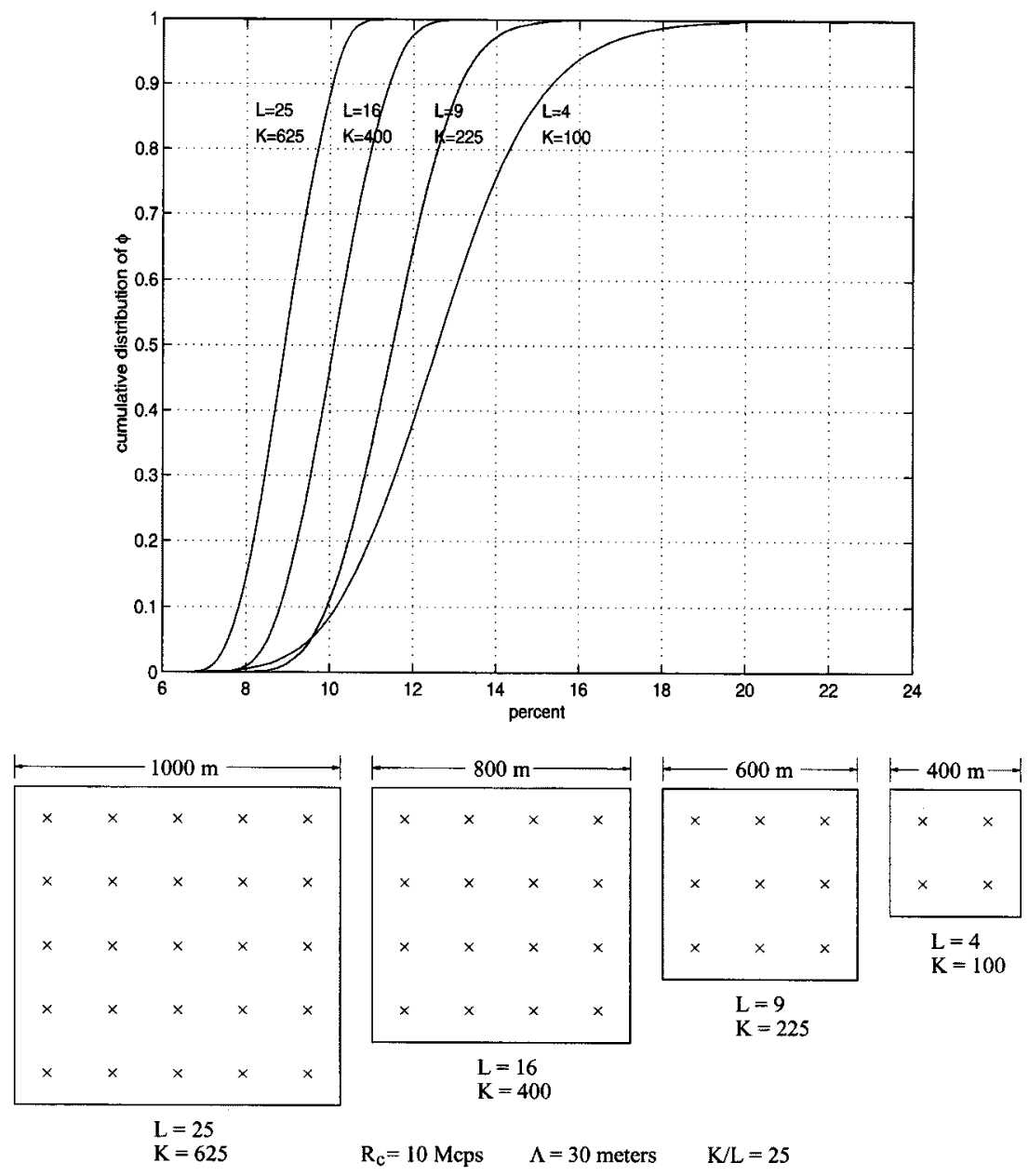

Fig. 19. Cumulative distribution function of $\phi$ that shows the effects of pooling the resources.

\section{Percent Correlation and Statistical Multiplexing}

Finally, in Fig. 19, the effects of pooling the resources are shown. In this figure, $\Lambda$ and $K / L$ are kept fixed at $30 \mathrm{~m}$ and 25 , respectively, and the size of the coverage region is changed in such a way that the inter-AE distance (or the number of AEs per unit area) is kept the same. It is observed from Fig. 19 that a higher $L$ and $K$ pair yields a lower $\phi$ value due to statistical multiplexing. Thus, if the maximum number of users a system with $L=4$ can accommodate is 100 , then a larger service region with $L=25$ (arranged in the way shown in Fig. 19) can actually accommodate more than 625 users.

\section{SUMMARY AND CONCLUSIONS}

It is reported in the literature that in the reverse link of a CDMA macrodiversity system a remarkable $L$-fold capacity increase can be attained by using $L$ AEs provided that the spread spectrum bandwidth approaches to infinity [1]. In a finite-bandwidth system, however, the increase in capacity as a result of the utilization of multiple AEs will be less than linear due to the presence of the correlated interference effects. In this paper, the effects of the system parameters on the severity of the spatial correlated interference are investigated. Further research is required for translating the correlated interference level into the actual loss in antenna gain (and thus in capacity).
We first analyzed the simplest nontrivial case of two AEs with two users, in order to develop some insight into the problem of correlated interference in the spatial domain. For this special case, we were able to obtain the correlation coefficient as a function of the distances involved in the service region. Hence, for a given user location, we were able to determine the portions of the service region in which other user(s) would cause correlated interference to the given user; we called this region the caution zone for the given user. We then found an approximate expression (and an approximate caution zone) in simpler terms.

Next, we investigated the most general case of many AEs and many users. Due to the computational complexity, however, we worked with a performance metric which we called percent correlation, instead of the correlation coefficient itself. The direct relation between the correlation coefficient and percent correlation is obvious.

The results presented indicate that the distance between the AEs in interference-limited CDMA macrodiversity systems can be compared against the parameter which we defined as the chiplength ([speed of light]/[chip rate]) of the spreading code. In this respect, it is observed that the inter-AE distance should be (many times) greater than the chiplength. It can be concluded that in systems where this condition is satisfied, close to linear antenna gains can be achieved with multiple AEs placed as far apart as possible in the service region. We emphasize that this 
TABLE I

NORMALIZED CROSS-CORRELATION PROPERTIES OF GOLD CODES

\begin{tabular}{l|l|l|c}
\hline $\begin{array}{l}\text { Shift-Register } \\
\text { Length, } p\end{array}$ & $\begin{array}{l}\text { Code Period } \\
\text { (Length), } N\end{array}$ & $R_{12}(q) / \tau$ & $\begin{array}{c}\text { Approximate } \\
\text { Frequency of Occurrence }\end{array}$ \\
\hline$p$ : odd & $N=2^{p}-1$ & -1 & 0.50 \\
& & $-2^{(p+1) / 2}-1$ & 0.25 \\
& & $2^{(p+1) / 2}-1$ & 0.25 \\
\hline$p$ : even and not & $N=2^{p}-1$ & -1 & 0.75 \\
divisible by 4 & & $-2^{(p+2) / 2}-1$ & 0.125 \\
& & $2^{(p+2) / 2}-1$ & 0.125 \\
\hline
\end{tabular}

antenna gain achieved against interference is in addition to all types of diversity gains. If, on the other hand, the service region size is not large enough to enable such a separation between the AEs, then the returns in capacity (due to the antenna gain) will not be as high.

Since the chiplength is inversely related to the spread spectrum bandwidth, a wider bandwidth will enable the efficient utilization of multiple AEs in a macrodiversity system, in addition to wideband CDMAs many other benefits reported in the literature (such as, the finer resolution of the multipath components).

How often should a wireless signal be collected? It is important to determine a practical figure for the number of AEs to be placed in a given service region; such a figure should constitute a practical operating point given the law of diminishing returns with the increasing number of AEs and the associated complexity. The main result of this paper, the idea of comparing the inter-AE distance (which is determined by the size of the coverage region) against the chiplength (which is determined by the spread spectrum bandwidth), constitutes a step toward answering this question. Our hope is that further research in this area will result in a "spatial sampling theorem."

\section{APPENDIX A \\ GOLD CODES}

In Section II, correlation coefficient is obtained for Bernoulli spreading codes. In this section, a similar analysis is presented for Gold codes.

Let $R_{12}(q) / \tau$ be the normalized cross-correlation between the spreading codes of $w_{1}$ and $w_{2}$ (refer to (2) for the definition of $R_{12}(q)$ ). The three-level normalized cross-correlation properties of Gold codes is given in Table I [23, pp. 607-609].

We model the normalized cross-correlation as an independent three-valued discrete random variable with probability values equal to the corresponding frequencies of occurrence given in Table I. In this case, it is straightforward to show that

$$
\begin{aligned}
\mathbf{E}\left(\frac{R_{12}(q)}{\tau}\right) & =-1, \forall q \\
\mathbf{E}\left(\frac{R_{12}^{2}(q)}{\tau^{2}}\right) & =2^{p}+1=N+2, \forall q \\
\mathbf{E}\left(\frac{R_{12}(q) R_{12}(r)}{\tau^{2}}\right) & =1, q \neq r
\end{aligned}
$$

Equations (37)-(39) are valid for all $p$ (shift-register length) values which are odd, or even and not divisible by 4 . It is observed comparing (37)-(39) with (12) that the probabilistic cross-correlation properties of Gold codes are not too different from those of Bernoulli codes.

It follows from (10), (38), and (39), that when Gold codes are used, (40)-(41) is obtained, as shown at the bottom of the page.

Now, $\rho_{12, I-I I}$ for Gold codes can be obtained by inserting (40) and (41) in (11). In the $\mathbf{E}\left(\eta_{12 j}^{2}\right)$ and $\mathbf{E}\left(\eta_{12 I} \eta_{12 I I}\right)$ expressions given in (40) and (41), respectively, the terms with $(N+2) \tau^{2}$ factor will dominate when compared to the terms with only $\tau^{2}$ factor, for large $N$. It then follows that the correlation coefficient for Gold codes will asymptotically approach to that for Bernoulli codes as $N$ increases. Therefore, although (14) which holds for Bernoulli codes is not valid anymore for Gold codes due to (39) (i.e., although $\rho_{12, I-I I}$ will be nonzero for the entire $t_{12 I}$ - $t_{12 I I}$ plane for Gold codes), the value of $\rho_{12, I-I I}$ outside the region given by (14) will approach to zero asymptotically as $N$ increases. In Fig. 4, $\rho_{12, I-I I}$ is plotted for Gold codes with $N=127(p=7)$ for various values of $t_{12 I}$, in the range of $[0,1]$ and for $-1 \leq t_{12 I I} \leq 2$. Comparing Figs. 3 and 4 , we conclude that the correlation coefficient analysis presented in Section II with Bernoulli codes can be considered as a close approximation of a similar analysis with the more realistic Gold codes as long as $N$ is not small.

$$
\begin{aligned}
& \mathbf{E}\left(\eta_{12 j}^{2}\right)=(N+2) \tau^{2}\left[\left(t_{12 j}-\left\lfloor t_{12 j}\right\rfloor\right)^{2}+\left(\left\lceil t_{12 j}\right\rceil-t_{12 j}\right)^{2}\right]+2 \tau^{2}\left(t_{12 j}-\left\lfloor t_{12 j}\right\rfloor\right)\left(\left\lceil t_{12 j}\right\rceil-t_{12 j}\right) \\
& \mathbf{E}\left(\eta_{12 I} \eta_{12 I I}\right)=\left\{\begin{array}{l}
\tau^{2}\left(t_{12 I}-\left\lfloor t_{12 I}\right\rfloor\right)\left(t_{12 I I}-\left\lfloor t_{12 I I}\right\rfloor\right)+\tau^{2}\left(t_{12 I}-\left\lfloor t_{12 I}\right\rfloor\right)\left(\left\lceil t_{12 I I}\right\rceil-t_{12 I I}\right) \\
+\tau^{2}\left(\left\lceil t_{12 I}\right\rceil-t_{12 I}\right)\left(t_{12 I I}-\left\lfloor t_{12 I I}\right\rfloor\right)+\tau^{2}\left(\left\lceil t_{12 I}\right\rceil-t_{12 I}\right)\left(\left\lceil t_{12 I I}\right\rceil-t_{12 I I}\right), \\
\text { for }\left\lfloor t_{12 I}\right\rfloor<\left\lfloor t_{12 I I}\right\rfloor-1, \text { or }\left\lceil t_{12 I}\right\rceil>\left\lceil t_{12 I I}\right\rceil+1 ; \\
(N+2) \tau^{2}\left(t_{12 I}-\left\lfloor t_{12 I}\right\rfloor\right)\left(t_{12 I I}-\left\lfloor t_{12 I I}\right\rfloor\right)+\tau^{2}\left(t_{12 I}-\left\lfloor t_{12 I}\right\rfloor\right)\left(\left\lceil t_{12 I I}\right\rceil-t_{12 I I}\right) \\
+\tau^{2}\left(\left\lceil t_{12 I}\right\rceil-t_{12 I}\right)\left(t_{12 I I}-\left\lfloor t_{12 I I}\right\rfloor\right)+(N+2) \tau^{2}\left(\left\lceil t_{12 I}\right\rceil-t_{12 I}\right)\left(\left\lceil t_{12 I I}\right\rceil-t_{12 I I}\right), \\
\text { for }\left\lfloor t_{12 I}\right\rfloor=\left\lfloor t_{12 I I}\right\rfloor ; \\
\tau^{2}\left(t_{12 I}-\left\lfloor t_{12 I}\right\rfloor\right)\left(t_{12 I I}-\left\lfloor t_{12 I I}\right\rfloor\right)+\tau^{2}\left(t_{12 I}-\left\lfloor t_{12 I}\right\rfloor\right)\left(\left\lceil t_{12 I I}\right\rceil-t_{12 I I}\right) \\
+(N+2) \tau^{2}\left(\left\lceil t_{12 I}\right\rceil-t_{12 I}\right)\left(t_{12 I I}-\left\lfloor t_{12 I I}\right\rfloor\right)+\tau^{2}\left(\left\lceil t_{12 I}\right\rceil-t_{12 I}\right)\left(\left\lceil t_{12 I I}\right\rceil-t_{12 I I}\right), \\
\text { for }\left\lfloor t_{12 I}\right\rfloor=\left\lceil t_{12 I I}\right\rceil ; \\
\tau^{2}\left(t_{12 I}-\left\lfloor t_{12 I}\right\rfloor\right)\left(t_{12 I I}-\left\lfloor t_{12 I I}\right\rfloor\right)+(N+2) \tau^{2}\left(t_{12 I}-\left\lfloor t_{12 I}\right\rfloor\right)\left(\left\lceil t_{12 I I}\right\rceil-t_{12 I I}\right) \\
+\tau^{2}\left(\left\lceil t_{12 I}\right\rceil-t_{12 I}\right)\left(t_{12 I I}-\left\lfloor t_{12 I I}\right\rfloor\right)+\tau^{2}\left(\left\lceil t_{12 I}\right\rceil-t_{12 I}\right)\left(\left\lceil t_{12 I I}\right\rceil-t_{12 I I}\right), \\
\text { for }\left\lceil t_{12 I}\right\rceil=\left\lfloor t_{12 I I}\right\rfloor .
\end{array}\right.
\end{aligned}
$$




\section{APPENDIX B \\ THE HYPERBOLIC GRID}

In this section, a systematic way of finding the approximate caution zones on the unit service region will be presented. We assume that $\mathrm{AE}$ locations are fixed and given.

For a given $w_{1}$ location, we calculate $h_{1 I}-h_{1 I I}$ [refer to (18)] and denote this difference by $\vartheta_{1}$. Since "a hyperbola is the set of all points in a plane the difference of whose distances from two fixed points is a constant" [24, p. 595], for the given $w_{1}$ location, a hyperbola can be drawn for representing all the points which satisfy

$$
h_{1 I}-h_{1 I I}=\vartheta_{1}
$$

We note that for a given AE $I$ and $I I$ locations, there is only a unique hyperbola through the $w_{1}$ location.

For an arbitrary $w_{2}$ location, we similarly calculate $h_{2 I}-h_{2 I I}$ and denoted it by $\vartheta_{2}$. A corresponding hyperbola can also be drawn for representing all the points which satisfy

$$
h_{2 I}-h_{2 I I}=\vartheta_{2} \text {. }
$$

The importance of the expression $\Delta t_{12, I-I I}$ has been discussed in Section III; now, we will express this expression in terms of $\vartheta_{1}$ and $\vartheta_{2}$. From (17), (18), (26), (42) and (43), $\Delta t_{12, I-I I}$ can be written as

$$
\begin{aligned}
\Delta t_{12, I-I I} & =\frac{s R_{c}}{c}\left[\left(h_{1 I}-h_{1 I I}\right)-\left(h_{2 I}-h_{2 I I}\right)\right] \\
& =\frac{s R_{c}}{c}\left(\vartheta_{1}-\vartheta_{2}\right) .
\end{aligned}
$$

We emphasize that $w_{1}$ location is given and our goal is to find the approximate caution zone for $w_{1}$; in other words, $\vartheta_{1}$ is known but $\vartheta_{2}$ is to be found. For a given $w_{1}$ location, we first compute $\vartheta_{1}$ from (42). Next, in order to find the boundaries of the approximate caution zone, we solve the equation [refer to (30)]

$$
\frac{s R_{c}}{c}\left(\vartheta_{1}-\vartheta_{2}\right)= \pm 1
$$

for $\vartheta_{2}$ and denote the two solutions as $\vartheta_{21}$ and $\vartheta_{22}$. Then, using (43), we draw the hyperbolas corresponding to $\vartheta_{21}$ and $\vartheta_{22}$. The approximate caution zone is the area between these two hyperbolas.

For the exemplary system given in Section II-B, these two hyperbolas and the approximate caution zone for $w_{1}$ are shown in Fig. 11. In this figure, all the hyperbolas for which $\left(\vartheta_{1}-\right.$ $\left.\vartheta_{2}\right) s R_{c} / c$ is an integer are also shown; we call this set of hyperbolas the hyperbolic grid. On each hyperbola in the hyperbolic grid, the corresponding $\left(\vartheta_{1}-\vartheta_{2}\right) s R_{c} / c$ value is written; it is observed from (44) that these integers are indeed $\Delta t_{12, I-I I}$ values. We note that the "origin" of the hyperbolic grid is the hyperbola on which $w_{1}$ is located.

It is worth emphasizing that in Fig. 11, if $w_{1}$ were not at the given location but were at some other location on the same hyperbola which satisfies $h_{1 I}-h_{1 I I}=\vartheta_{1}$ (that is, the hyperbola indicated by " 0 " in Fig. 11), the locations of the other hyperbolas and the approximate caution zone would still be the same. Similarly, as far as the correlation analysis is concerned, as long as $w_{2}$ is on a particular hyperbola, it does not matter where it is on that hyperbola.

\section{REFERENCES}

[1] S. V. Hanly, "Capacity and power control in spread spectrum macrodiversity radio networks," IEEE Trans. Commun., vol. 44, no. 2, pp. 247-256, Feb. 1996

[2] H. Yanikomeroglu and E. S. Sousa, "CDMA sectorized distributed antenna system," in Proc. IEEE Int. Symp. Spread Spectrum Techniques and Applications (ISSSTA'98), Sun City, South Africa, Sept. 1998, pp. 792-797.

[3] D. Tang, "Fiber-optic antenna remoting for multisector cellular cell sites," in Proc. IEEE Int. Conf. Commun. (ICC'92), vol. 1, 1992, pp. $76-81$.

[4] M. Shibutani, T. Kanai, W. Domon, K. Emura, and J. Namiki, "Optical fiber feeder for microcellular mobile communication system (H-015)," IEEE J. Select. Areas Commun., vol. 11, pp. 1118-1126, Sept. 1993.

[5] H. Yanikomeroglu and E. S. Sousa, "Antenna interconnection strategies for personal communication systems," IEEE J. Select. Areas Commun., vol. 15/7, pp. 1327-1336, Sept. 1997.

[6] J. Zander, "Performance of optimum transmitter power control in cellular radio systems," IEEE Trans. Veh. Technol., vol. 41, pp. 57-62, Feb. 1992.

[7] H. Yanikomeroglu and E. S. Sousa, "SIR-balanced macro power control for CDMA sectorized distributed antenna systems," in Proc. IEEE 9th Int. Symp. Personal, Indoor and Mobile Radio Commun. (PIMRC'98), Boston, USA, Sept. 1998.

[8] M. Andersin, Z. Rosberg, and J. Zander, "Distributed discrete power control in cellular PCS," Wireless Personal Commun., vol. 6, no. 3, 1998.

[9] H. Yanikomeroglu and E. S. Sousa, "On the power control and number of antenna elements in CDMA distributed antenna system," in Proc. IEEE Int. Conf. Commun. (ICC'98), Atlanta, GA, June 1998, pp. 1040-1045.

[10] A. Obaid and H. Yanikomeroglu, "Reverse-link power control in CDMA distributed antenna systems," in Proc. IEEE Wireless Commun. and Networking Conf. (WCNC'00), Chicago, IL, Sept. 2000.

[11] R. C. Bernhardt, "Macroscopic diversity in frequency reuse radio systems," IEEE J. Select. Areas Commun., vol. 5, pp. 862-870, June 1987.

[12] W. Lee, "Smaller cells for greater performance," IEEE Commun. Mag., vol. 29, no. 1, pp. 19-30, 1991.

[13] J. H. Winters, J. Salz, and R. D. Gitlin, "The impact of antenna diversity on the capacity of wireless communication systems," IEEE Trans. Commun., vol. 42, pp. 1740-1751, Feb.-Apr. 1994.

[14] J. Proakis, Digital Communications, 4th ed. New York: McGraw-Hill, 2001

[15] T. S. Rappaport, Wireless Communications: Principles \& Practice. Upper Saddle River, NJ: Prentice-Hall, 1999.

[16] A. J. Viterbi, CDMA: Principles of Spread Spectrum Communications: Addison-Wesley, 1995.

[17] Z. J. Haas and C.-P. Li, "The multiply-detected macrodiversity scheme for wireless systems," IEEE Trans. Veh. Technol., vol. 47, pp. 506-530, May 1998.

[18] M. Schwartz, W. R. Bennett, and S. Stein, Communication Systems and Techniques. New York: McGraw-Hill, 1966.

[19] S. V. Hanly and D. N. C. Tse, "Resource pooling and effective bandwidth in CDMA networks with multiuser receivers and spatial diversity," IEEE Trans. Inform. Theory, vol. 47, pp. 1328-1351, May 2001.

[20] J. H. Winters, "Optimum combining in digital mobile radio with co-channel interference," IEEE J. Select. Areas Commun., vol. 2, July 1984.

[21] W. C. Jakes, Microwave Mobile Communications, W. C. Jakes, Ed. New York: Wiley, 1974.

[22] H. Yanikomeroglu and E. S. Sousa, "Correlated interference analysis in CDMA multi-antenna systems," in Proc. IEEE Int. Conf. on Commun. (ICC'99), Vancouver, Canada, June 1999, pp. 23-28.

[23] S. Haykin, Communication Systems, 3rd ed. New York: Wiley, 1994.

[24] R. A. Silverman, Calculus With Analytic Geometry. Englewood Cliffs, NJ: Prentice-Hall, 1985. 


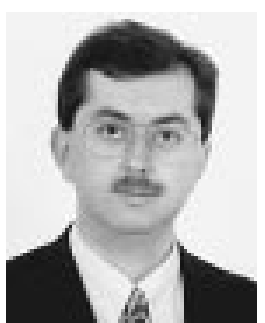

Halim Yanikomeroglu (S'97-M'99) was born in Giresun, Turkey, in 1968. He received his B.Sc. degree in electrical and electronics engineering from the Middle East Technical University, Turkey, in 1990 and his M.A.Sc. and Ph.D. degrees in electrical and computer engineering from the University of Toronto in 1992 and 1998, respectively.

$\mathrm{He}$ was with Marconi Kominikasyon A.S., Ankara, Turkey, from January 1993 to July 1994. Since 1998 he has been with the Department of Systems and Computer Engineering at Carleton University, Ottawa, where he is now a tenured Assistant Professor. His research interests include almost all systems aspects of wireless communications with a special emphasis on cellular multihop networks, radio resource management and CDMA multi-antenna systems. At Carleton University, he teaches graduate courses on digital, mobile and wireless communications. He has been involved in the technical program committees of numerous international conferences in wireless communications; he has also given several tutorials in such conferences.

Dr. Yanikomeroglu is currently serving as the Secretary of the IEEE Technical Committee on Personal Communications. He is a Registered Professional Engineer in the Province of Ontario, Canada.

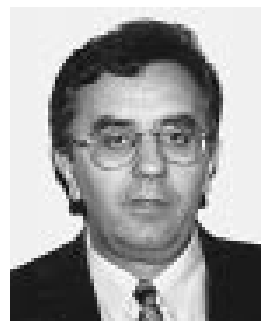

Elvino S. Sousa (S'82-M'86-SM'96) received his B.A.Sc. in engineering science and the M.A.Sc. in Electrical Engineering from the University of Toronto in 1980 and 1982 respectively and his Ph.D. in electrical engineering from the University of Southern California in 1985. Since 1986 he has been with the department of Electrical and Computer Engineering at the University of Toronto where he is now a Professor and BUL Chair in Computer Engineering. He has performed research in spread spectrum systems and CDMA since 1983. His current interests are in the areas of high-speed CDMA systems, smart antenna systems, software radio, ad-hoc networks and wireless system concepts for 4th generation networks. At the University of Toronto he is the director of the wireless lab, which has undertaken research in CDMA wireless systems for the past 15 years. He has been invited to give lectures and short courses on spread spectrum, CDMA and wireless communications in a number of countries and has been a consultant to industry and Governments in the area of wireless systems internationally.He was the technical program chair for PIMRC'95 and vice-technical program chair for Globecom' 99 and has been involved in the technical program committee of numerous international conferences. He was the past chair of the IEEE Technical committee on Personal Communications. He has spent sabbatical leaves at Qualcomm and Sony CSL/ATL, where he was the holder of the Sony sabbatical chair. Currently he is the holder of the Bell University Labs Chair in Computer Engineering with a mandate for research in wireless computing and the principal investigator in the BUL Mobile Computing Lab. 